FACULDADE DE CIÊNCIAS ECONô MICAS dA UFRGS
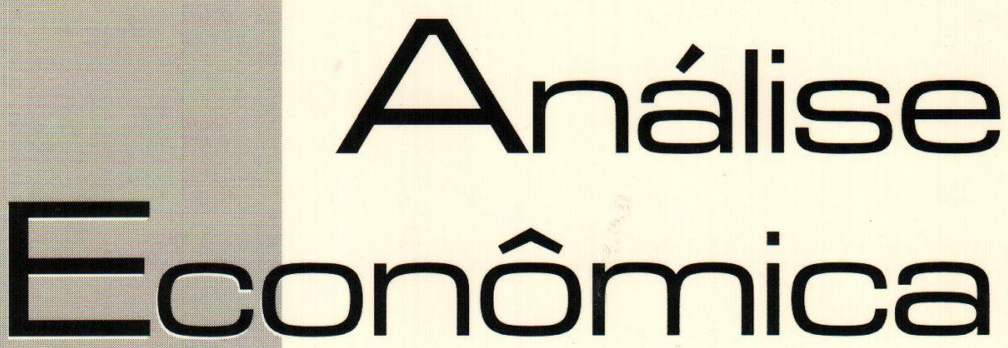

ESCOLHA DE PORTFÓLIO, INVESTIMENTO E NĀO. NEUTRALIDADE DA MOEDA

JOSÉ LUIS OREIRO

REFORMAS NA AROUITETURA FINANCEIRA INTERNACIONAL: NOVIDADES NO FRONT? ANDRÉ MOREIRA CUNHA

MERCOSUR'S CHANGE IN TRADE PATTERNS ANDRÉ FILIPE ZAGO DE AZEVEDO

O IMPACTO DA COMPOSICÃO SETORIAL, DOS FLUXOS INTRA-SETORIAIS E DA ABERTURA COMERCIAL NA PARTICIPAÇÁO DE MERCADO DAS EXPORTAÇÓES BRASILEIRAS

CLÉSIO LOURENÇO XAVIER E EMERSON FERNANDES MARÇAL

O EFEITO BALASSA-SAMUELSON E A PARIDADE DO PODER DE COMPRA NA ECONOMIA BRASILEIRA CLÁUDIO ROBERTO FÓFFANO VASCONCELOS

CICLOS Y FLUCTUACIONES FINANCIERAS: LA IRREGULAR DINÁMICA ECONÓMICA

SARY LEVY-CARCIENTE

O PENSAMENTO DE KARL POPPER: AS DIFERENTES INTERPRETACÓES DOS METODÓLOCOS DA CIENCIA ECONÓMICA

SOLANCE REGINA MARIN E RAMÓN GARCÍA FERNÁNDEZ

A EXPANSÃO DO ESCOPO TEMÁTICO DAS NEGOCIAÇÓES COLETIVAS DE TRABALHO CARLOS HENRIQUE HORN

EFEITOS DO CAPITAL SOCIAL E DO CAPITAL POLITICO NO DESENVOLVIMENTO ECONOMMICO SIMULAÇOES PARA PAISES E ESTADOS BRASILEIROS

RONALDO A. ARRAES, RICARDO CANDÉA S. BARRETO E VLADIMIR KÜHL TELES

O PROBLEMA DE RISCO MORAL NO MERCADO BRASILEIRO DE ASSISTÊNCIA MÉDICA SUPLEMENTAR LUCIANA PINTO DE ANDRADEE SABINO DA SILVA PÓRTO JÚNIOR

ANO

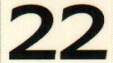

No 41

Março, 2004 
Universidade Federal do Rio Grande do Sul

Reitora: Profo. Wrana Maria Panizzi

Faculdade de Ciencias ECONOMICAS

Diretora: Prof Pedro César Dutra Fonseca

Centro de Estudos e Pesquisas Economicas

Diretor: Prof. Gentil Corazza

Departamento de Ciencias Económicas

Chefe: Prof. Ricardo Dathein

Curso de Pós-Graduação em Economia

Coordenador: Prof. Eduardo Pontual Ribeiro

Programa de Pó-Graduação em Desenvolvimento Rural

Coordenador: Prof. Jalcione Almeida

CONSElHo EDITORIAL:

Carlos G. A. Mielitz Netto (UFRGS), Eduardo A. Maldonado Filho (UFRGS), Eduardo P. Ribeiro (UFRGS), Eleutério F. S. Prado (USP), Eugênio Lagemann (UFRGS), Fernando Cardim de Carvalho (UFRJ), Fernando Ferrari Filho (UFRGS), Fernando de Holanda Barbosa (FGV/RJ), Flávio Vasconcellos Comim (UFRGS), Gentil Corazza (UFRGS), Giácomo Balbinotto Netto (UFRGS), Gustavo Franco (PUC/RJ), Jan A. Kregel (UNCTAD), João Rógério Sanson (UFSC), Joaquim Pinto de Andrade (UnB), Jorge Paulo Araújo (UFRGS), Juan H. Moldau (USP), Marcelo S. Portugal (UFRGS), Maria Alice Lahorgue (UFRGS), Paul Davidson (University of Tennessee), Paulo D. Waquil (UFRGS), Pedro C. D. Fonseca (UFRGS), Philip Arestis (Levy Economics Institut of Bard College), Roberto C. de Moraes (UFRGS), Ronald Otto Hillbrecht (UFRGS), Sabino da Silva Porto Jr. (UFRGS), Stefano Florissi (UFRGS) e Werner Baer (University of Illinois at Urbana-Champaign).

COMISSĀO EDITORIAL:

Eduardo Augusto Maldonado Filho, Fernando Ferrari Filho, Gentil Corazza, Marcelo Savino Portugal, Paulo Dabdab Waquil e Roberto Camps Moraes.

EDITOR: Prof. Fernando Ferrari Filho

Editor Adjunto: Prof. Gentil Corazza

SeCretária: Paulo Roberto Eckert

REVISÃO DE TEXTOS: Vanete Ricacheski

EdITORAÇÃo Eletrónca: Vanessa Hoffmann de Quadros

Fundador: Prof. Antônio Carlos Santos Rosa

Os materiais publicados na revista Análise Econômica são da exclusiva responsabilidade dos autores. É permitida a reprodução total ou parcial dos trabalhos, desde que seja citada a fonte. Aceita-se permuta com revistas congêneres. Aceitam-se, também, livros para divulgação, elaboração de resenhas e recensões. Toda correspondência, material para publicaçāo (vide normas na terceira capa), assinaturas e permutas devem ser dirigidos ao seguinte destinatário:

PROF. FERNANDO FERRARI FILHO Revista Análise Econômica - Av. João Pessoa, 52 CEP 90040-000 PORTO ALEGRE - RS, BRASIL Telefones: (051) 316-3513 - Fax: (051) 316-3990

Análise Econômica E-mail: rae@ufrgs.br

Ano 22, $n^{\circ} 41$, março, 2004 . Porto Alegre

Faculdade de Ciências Econômicas, UFRGS, 2004

Periodicidade semestral, março e setembro.

Tiragem: 500 exemplares

1. Teoria Econômica - Desenvolvimento Regional -

Economia Agrícola - Pesquisa Teórica e Aplicada -

Periódicos. I. Brasil

Faculdade de Ciências Econômicas,

Universidade Federal do Rio Grande do Sul. 


\title{
Ciclos y Fluctuaciones Financieras: La Irregular Dinámica Económica
}

\author{
Sary Levy-Carciente \\ (IIES-FACES-UCV, econofin@cantv.net)
}

\begin{abstract}
Síntesis: Desde una perspectiva postkeynesiana y que toma en cuenta las fluctuaciones como mecanismo endógeno y dinámico del comportamiento económico, se desarrolla un modelo que permite mostrar cómo movimientos cíclicos de determinadas variables, producidos como consecuencia de las fluctuaciones de la economía, devienen en dinámicas marcadamente irregulares. Las dinámicas resultantes impiden que políticas derivadas de esquemas convencionales, lineales, atemporales y con excesiva simplificación tengan los efectos equilibrantes que buscan.
\end{abstract}

Palabras Claves: Fluctuaciones, Inestabilidad Financiera, Dinámica NoLineal, Irregularidad, Fragilidad financiera.

\begin{abstract}
From a postkeynessian perspective, that takes into account fluctuations as endogenous and dynamic mechanism of the economic behavior, we develop a model that allows us to show how cyclical movements of certain variables, taken place as consequence of the fluctuations of the economy, become in a highly irregular dynamic. The resulting dynamics impede that policies derived from conventional, linear, not-time-related perspectives or with excessive simplification, could achieve the equilibrium they look for:
\end{abstract}

Key-words: fluctuations, financial instability, non-linear dynamic, irregularity, financial fragility.

JEL: E32, E6.

\section{Introducción}

La inestabilidad y las continuas crisis financieras acaecidas desde finales de los $80^{\prime}$ son indicios de la necesidad de profundizar en el estudio de aquellas situaciones alejadas del equilibrio, es decir, en los periodos de crisis y sus desencadenantes. Asimismo, la escalada en las crisis pareciera indicar algún mecanismo que incrementa los niveles de inestabilidad, lo que podría ser indicio de una tendencia hacia escenarios no convergentes. 
Lo señalado con anterioridad lleva a considerar una visión que tome en cuenta las fluctuaciones como mecanismo endógeno y dinámico del comportamiento económico. Las fluctuaciones o ciclos económicos son el resultado del proceso de acumulación del sistema capitalista y en tanto que la acumulación depende principalmente de la inversión, ésta será la variable macroeconómica en la que se focalizará el análisis. Además, tomando en cuenta el natural vínculo entre los niveles micro y macroeconómico y las esferas real y financiera de la economía, se hace necesario revisar las características de la demanda para la inversión y por ende de sus fuentes de financiamiento.

Para su exposición el escrito se desarrolla como sigue: se destacan perspectivas teóricas relevantes sobre los ciclos endógenos, se prosigue con la revisión de algunos aportes para el estudio de las fluctuaciones de la inversión que vinculan las esferas real y nominal de la economía, se desarrolla un modelo que emula las fluctuaciones por medio de transformadas trigonométricas evaluando diversas situaciones y finalmente se presentan las reflexiones del trabajo.

\section{Ciclos y Fluctuaciones}

El énfasis de los teóricos clásicos en el equilibrio tiene su raíz en la llamada Ley de Say - Toda oferta crea su demanda - enfatizada por la Mano Invisible de Adam Smith - que permitía al mercado el logro del equilibrio. De ahí que encarguen al sector privado de los procesos de producción y distribución y releguen al Estado los de defensa y seguridad nacional y emisión monetaria. Gradualmente, la Ley de Say fue refinada por diversos teóricos, entre ellos Mill, Marshall, Jevons y Walras, hasta configurarse en el principio de la flexibilidad de los precios que permite el ajuste automático de los mercados y que faculta a la economía situarse en su nivel de pleno empleo.

Pero la experiencia, lejos de equilibrio, lo que nos muestra son procesos de cambio, con períodos de auge y crisis y los acercamientos explicativos a estos procesos han sido variados.

Siguiendo a Marx (1977 [1867]), la base del sistema capitalista es la acumulación, la cual es posible gracias al proceso de reproducción ampliada del capital, el cual genera un incremento del capital cons- 
tante por sobre el capital variable, que a la larga conlleva a un descenso gradual de la cuota general de la ganancia ${ }^{1}$. Al disminuir la cuota de ganancia, aumenta el mínimo de capital que se requiere para el proceso de producción, tendiéndose a un aumento de la concentración del capital, que nuevamente presiona a la baja la tasa de la ganancia ${ }^{2}$. Así, se explica el origen de la crisis congénita del sistema capitalista, la cual es considerada inevitable y que finalmente llevaría al sistema a su autodestrucción.

Mitchell (1913), quien demostrara que la formulación de la ley de Say ignoraba la especificidad histórica de las instituciones, fue uno de los primeros en analizar el ciclo desde una perspectiva endógena $^{3}$. Señala que los ciclos comienzan en Inglaterra (1793), siguiéndole los EUA (1796), Francia (1847), Alemania (1857), (18881891) la Rusia Zarista, Argentina, Brasil, Canadá, Sur África, Australia, India, Japón y China. A partir de 1890 los ciclos se hacen internacionales y así sus influencias. En su estudio muestra como el ciclo se produce como consecuencia del desenvolvimiento normal de los negocios, en un ambiente institucional dado. Gran parte del trabajo de establecer la periodicidad de los ciclos se debe a este autor.

Por su parte, Keynes (1936) nos indica el rol determinante de variación de la eficiencia marginal del capital en los desequilibrios sucedidos en la economía. El argumento puede resumirse como sigue: en períodos de crecimiento el desempleo disminuye, la capacidad de demanda se incrementa, por lo que se estimula la inversión. Esta situación se retroalimenta positivamente produciendo salarios monetarios altos que conllevan a una reducción de la eficiencia marginal del capital, por lo comienza a reducirse el estímulo a la inversión. En este escenario, la crisis al incrementar el desempleo, presiona a la baja los niveles salariales, restableciendo la tasa de beneficio. Pero además de tomar en cuenta el efecto del crecimiento del capital en el largo, destaca su influencia en el corto plazo, al analizar la determinación de la demanda agregada, haciendo énfasis en la incertidumbre y los aspectos monetarios financieros en la determinación de la inversión. La explicación keynesiana de los ciclos la refiere como parte de la naturaleza propia del sistema, por

\footnotetext{
I Tomo III, Sección III, Cap XIII, :214.

${ }^{2}$ Tomo III, Sección III, Cap XV: 246 y ss.

${ }^{3}$ Aunque durante el siglo anterior ya lo habia hecho Juglar.
} 
ende es de carácter endógeno y en tanto que generadora de inestabilidad, promueve la intervención para disminuir los impactos negativos de los períodos de crisis:

"... i el remedio del auge no es una tasa más alta de interés, sino una más baja!; porque ésta puede hacer que perdure el llamado auge. El remedio correcto para el ciclo económico no puede encontrarse en evitar los auges y conservarnos así en semi-depresiones permanentes, sino en evitar las depresiones y conservarnos de este modo en un cuasi-auge continuo" (Keynes, 1980 [1936]:286).

Schumpeter (1939) elabora una explicación tomando en cuenta el impacto de las innovaciones tecnológicas en la economía que alterarían la regularidad de la actividad bancaria, por lo que no se deslinda totalmente de las explicaciones exógenas de los ciclos:

"El ciclo de los negocios que nos concierne... no es análogo a la oscilación de una cuerda elástica... que una vez puesta en movimiento, si no fuera por la fricción, seguiría indefinidamente... (los ciclos no se auto-reproducen, sino que) son debidas a la intermitente acción de las fuerzas de la innovación ${ }^{4 "}$

Considera que la expansión económica comienza cuando la innovación es utilizada por un empresario en su industria, quien es seguido por otros en la competencia. El auge finaliza tan pronto el ímpetu de la innovación se acaba. Schumpeter se concentra en los ciclos de Kondratieff (30 a 50 años) aunque trató también de explicar ciclos más cortos 5 . En lo que nos compete, la parte más interesante de planteamiento de Schumpeter es la incorporación de tres elementos: cambios endógenos a partir del análisis del flujo circular incorporando una perspectiva dinámica frente a la tradicional estática o estática comparativa, la inclusión del crédito en el análisis económico y la incorporación de conceptos monetarios de capital

\footnotetext{
${ }^{4}$ Traducción propia de: "The business cycles with which we are concerned ... are not analogous to the oscillation of an elastic string membrane ... which, once set into motion, would but for friction, go on indefinitely ... [cycles are not self-generating, but] are due to the intermittent action of the "force' of innovation" (Schumpeter, 1939:175)

${ }^{5}$ Existen diversas formas de establecer la periodicidad para evaluar los ciclos. Además de los ciclos de Kondratieff están: el ciclo de Kutznet (por Simon Kutznets), de 15 años, asociado a los ciclos de la industria de la construcción; el ciclo de Juglar (por Clement Juglar) de 10 años, asociado a las profundas depresiones sucedidas en el s19 producto de la caída de las inversiones en plantas y equipos y el ciclo de Kitchin (por Joseph Kitchin) que tiene una duración de 3-4 años, y son frecuentemente asociados a variación en los inventarios.
} 
e interés en reemplazo de conceptos 'reales'. Sin embargo no logró 'traer al dinero a la base ${ }^{61}$ en su análisis (Earley, 1997:340).

Posteriormente, Kalecki (1965) formaliza un modelo inherentemente cíclico partiendo de un mercado oligopólico que le permite aflorar el conflicto capital / trabajo. Considera los cambios en la función de inversión (en especial el acelerador) y la interacción de los mecanismos de formación de precios y de salarios (considerados inflexibles) como causas endógenas de los ciclos. Además anticipa el impacto de influencias externas, tales como, cambio en los precios externos, cambio en la demanda externa y la aparición de nuevas tecnologías que mantendrían a la economía en continuo oscilar. De lo anterior deriva que la presencia de ciclos es una característica propia del sistema capitalista, aunque los mismos no se presenten de forma regular.

En la tradición postkeynesiana, Hyman Minsky (1982) construye un modelo que explica las fluctuaciones económicas como el resultado de la especulación, la inversión y la deuda financiera en un entorno marcado por la incertidumbre. El modelo, bajo un esquema de oferta monetaria endógena, le otorga un rol activo a los intermediarios financieros, quienes al tomar ventaja de las condiciones cambiantes del mercado, generan un ambiente de creciente fragilidad financiera. Así, en condiciones de crecimiento económico, se favorecen expectativas de inversión y por tanto el crédito que la viabiliza. En la medida que la prosperidad se incrementa, las expectativas optimistas se enfatizan, lo que presiona al alza el precio de los bienes de capital y el precio de las acciones de las empresas, las cuales finalmente terminan sobrevaluadas y sobre-endeudadas. La nueva situación transforma las expectativas en sentido pesimista: se comienzan a liquidar activos y comienza un período de deflación, esto conduce a una contracción de la inversión y finalmente de la demanda agregada.

Kindleberger (1996 [1978]), parte del modelo de Minsky y en una perspectiva de carácter histórico revela los patrones comunes presentes en las diversas crisis internacionales desde el s. XVIII. Señala que si bien estas crisis pudieron ser desencadenadas por el

' Traducción propia de: 'Bring the money to the ground floor'. 
desplazamientos ${ }^{7}$ de elementos de naturaleza diversa (causa próxi$\mathrm{ma}^{8}$ ), mantienen al carácter especulativo de la economía como su elemento en común (causa remota). Su explicación parte de un esquema de manía (exceso especulativo de carácter irracional), con expansión crediticia que lleva a la generación de burbujas (desviación de los valores fundamentales) y a un fracaso financiero, lo que desencadena un pánico generalizado (temor repentino y exceso de desconfianza) que presiona al crack (colapso del precio de los activos) y finalmente redunda en crisis (proceso que envuelve los dos anteriores). En lo que se aleja del modelo de Minsky es en que plantea que dadas las transformaciones estructurales e institucionales de la economía, tales como el desarrollo corporativo, la moderna banca y los avances en las comunicaciones, el modelo de Minsky no se ajusta a las actuales realidades.

Desde otras perspectivas disciplinarias, el fluctuar de la economía también ha sido motivo de interés por lo que ciertos autores incorporan elementos de análisis propios de la historia, la sociología, la geografía, la antropología y otras disciplinas.

Los autores de visión sistémica plantean un abordaje funcionalista de base cibernética, en el que sobresalen actores individuales, colectivos o institucionales, que toman opciones $\mathrm{y} / \mathrm{o}$ decisiones con relación a fines, objetivos o valores, definidos estos en términos pragmáticos y relacionados con la definición de posiciones, conquista de ventajas o afirmación de hegemonías.

Wallerstein (1991) parte de un trazado geo-histórico (precedido por Braudel) y refiere nuestro presente como un período de crisis, definida esta como un momento 'raro' en el cual las contradicciones internas de un sistema no le permiten continuar, por lo que considera la necesidad de la aparición de uno nuevo. Señala que la historia se constituye en un conjunto o sucesión de sistemas económicos mundiales, creando y recreando fronteras así como fragmentándolas o disolviéndolas. La unidad de análisis es el sistema histórico, que desde el s XVI define como un sistema-mundo-capitalista, el cual refiere fluctuaciones de largo plazo (ciclos de Kondratieff). El origen

\footnotetext{
7 Desplazamiento traduce a Displacement, definido como eventos que cambian la situación, extienden el horizonte y alteran las expectativas, impidiendo que las expectativas racionales fallen en la percepción de la situación y por ende en la respuesta a generar (Kindleberger, 1996 [1978]:29).

8 Ob cit: 92 .
} 
de los ciclos tiene en lo económico una raíz marxista, en tanto que es definida como consecuencia de las contradicciones del proceso de acumulación capitalista. Además destaca contradicciones que se presentan en el plano político como consecuencia de los movimientos anti-sistémicos que minan la capacidad del sistema de sobrevivir.

Arrighi (1998, 1999) enfatiza en la inestabilidad y adaptabilidad del mundo capitalista y el rol hegemónico de los estados en períodos de reorganización sistémica. Nos indica que si ampliamos nuestro horizonte temporal el peso del componente financiero que hoy presenciamos posee aspectos recurrentes: 'ciclos sistémicos de acumulación' cada uno de los cuales ha finalizado con crisis de sobre acumulación (la de la diáspora Genovesa, s. XVI; la de Holanda, nación proto-capitalista, s. XVIII; la de Gran Bretaña, imperio con redes informales de comercio, s. XIX; y ahora la de EUA, s. XX, estado-nación de dimensiones continentales con redes transnacionales de corporaciones y militares). Los ciclos sistémicos de acumulación son también etapas de formación y expansión gradual del sistema capitalista mundial, donde la crisis de cada ciclo marca el reemplazo de organizaciones territoriales y no territoriales, y la emergencia de nuevos tipos. Pero asimismo señala que estamos asistiendo a una profunda transición donde se dificulta la capacidad de predicción y que define como caos sistémico.

Lo que parece estar claro para muchos autores es la idea de que el sistema capitalista tiene un conjunto de características que, muy lejos de mantenerlo estable y en equilibrio, lo impulsan a alejarse de ese estado. Asimismo pareciera ser que a pesar de las diferencias de los elementos que caracterizan los ciclos, los mismos presentan un componente financiero tanto en su fase inicial como en su fase crítica y que este componente financiero no está en lo absoluto desligado de su correspondiente componente 'real'.

\section{De Lombard Street a Wall Street: Finanzas y Fluctuaciones.}

El impacto de la inversión en el producto y el empleo es determinante, tanto en el largo plazo, como en las fluctuaciones de la actividad económica en el corto plazo. El estudio de estas fluctuaciones cíclicas de la inversión ha sido tradicionalmente realizado a través de los modelos de 'dos precios'. 
Siguiendo a Kregel (1992) podemos señalar que como primer antecedente de estos modelos se tiene el planteamiento de Wicksell (1898) quien identifica la diferencia entre el interés real y el nominal como la fuente del proceso de acumulación, ya que manteniendo el último constante, ante un aumento del nivel de precios, el primero disminuye, lo cual genera una acumulación irreversible (asumiendo dinero y crédito como equivalentes). Posteriormente, Hawtrey (1913) señala que al modificarse el nivel de precios, cambia el costo del crédito influenciando la producción en un proceso que fluctúa. Por su parte, Fisher (1907) considerará que el incremento en el nivel de precios de la producción por sobre el nivel de precios de los activos financieros dependía del crecimiento de la oferta monetaria y su modelo será la base de los teóricos cuantitativos.

Keynes (1930) propone reemplazar la perspectiva cuantitativa del intercambio ${ }^{9}$ por una relación que midiera el nivel de precios de los bienes la producción y de los bienes de capital, señalando el rol del sistema bancario en la conformación de este último y de ahí, contribuir a las fluctuaciones de la inversión y finalmente del producto. Tomando en cuenta la estructura financiera (hoja de balance) del sector industrial y asumiendo que la inversión realizada es financiada por medio del sistema crediticio, el valor de los activos de capital real será equivalente a los pasivos financieros del sector. La adquisición de los activos de capital tomará en cuenta las expectativas del sector empresarial sobre sus precios, o lo que conocemos como la 'eficiencia marginal del capital'. Por su parte los pasivos financieros dependerán de las expectativas del público sobre el precio de la deuda, que no es otra cosa el interés, que identifica su 'preferencia por la liquidez'. Se observa, entonces, dos niveles en el modelo de dos precios de Keynes: el primero que distingue entre precios de la producción actual y precios de los bienes de capital; y el segundo, que distingue entre el precio de los activos de capital y el de los pasivos financieros (Kregel, 1992: 95).

Minsky (1982) parte de esta postura de dos niveles en el modelo de dos precios y muestra cómo la operación del sistema bancario produce fluctuaciones en los precios, que marcan los ciclos. Mientras el primer nivel permite mostrar la generación de la inestabilidad del

\footnotetext{
${ }^{9}$ Antes y simultáneamente a Keynes otros autores como Bernacer, Myrdal y Kalecki realizan una crítica a la teoría cuantitativa del dinero (Mata, 1999).
} 
proceso económico, el segundo muestra cómo un cambio en el precio de los activos afecta la confianza en del sistema bancario y puede generar pánicos financieros. Considerando la demanda agregada como conformada por consumo e inversión con sus respectivos precios formados endógenamente (precio de los bienes de consumo final, $\mathrm{Pi}$, y precio del capital y los activos financieros, $\mathrm{Pk}$ ), observamos que sus precios se forman de manera muy distinta. El precio de la producción actual estaría determinado, en el primer nivel del modelo, por la interacción de la oferta y la demanda tanto del producto como de la mano de obra, en un arreglo institucional dado. Este precio queda aislado del impacto directo del dinero. El precio de los activos de capital y los instrumentos financieros, se determina en el segundo nivel del modelo, y refleja las expectativas de los participantes del mercado, las cuales a siguiendo la tradición postkeynesiana, serán consideradas inciertas y no estarán sujetas a las leyes de la probabilidad. Así, el precio de los bienes de capital no estará determinado por sus costos de producción, sino por la relación entre la eficiencia marginal del capital y la preferencia por la liquidez. Desprendiéndose que, una alteración en la preferencia por la liquidez y por ende en la conformación de los portafolios individuales conllevaría a una transformación del precio de los bienes de capital $\mathrm{y}$ estos finalmente influirían en el precio de los bienes finales.

La interacción que se sucede entre los factores financieros y reales está claramente presente en la especificación que Minsky realiza de la estructura dinámica de las economías financieramente sofisticadas. Para este autor, las fluctuaciones y las crisis a las que están sometidas las economías son de carácter endógeno, existiendo una tendencia a la fragilidad financiera y la inestabilidad sistémica, potenciada por la sofisticación en los sistemas financieros y las políticas económicas para contrarrestar los $\operatorname{ciclos}^{10}$. El sistema permanecerá estable sólo si el flujo de ganancias permite a las empresas cubrir sus compromisos de deuda. De ahí que las expectativas futu-

\footnotetext{
${ }^{10}$ Así por ejemplo: ante una política monetaria restrictiva, la banca para incrementar sus ganancias genera innovación financiera, lo que presiona al alza la velocidad de circulación del dinero y por ende de las finanzas, disminuyendo el efecto de la disminución de la liquidez y por ende del ajuste, lo que a su vez favorece la contratación de créditos a bajo interés, y permite que el ratio deuda / ingreso se deteriore. De acá emerge la paradoja: la intervención gubernamental evita una depresión deflacionaria (se incrementa la innovación financiera y se bajan los costos del capital), pero al no darse ninguna corrección la inestabilidad se incrementa (lo que podría identificar la necesidad de un nuevo modelo o sistema).
} 
ras sobre ganancias futuras afecten el nivel de inversión y el grado de endeudamiento deseado. Una vez que la estructura financiera es frágil, la crisis puede desencadenarse y amplificarse ante cualquier shock que modifique las expectativas negativamente. De lo anterior se observa que para Minsky el proceso de acumulación y su financiamiento presenta dinámicas no-lineales, en tanto que períodos de estabilidad pueden dar origen a situaciones turbulentas e inestables. A pesar de lo interesante que pudiesen resultar los trabajos de Minsky, la falta de un modelo que resuma sus planteamientos ha sido impedimento para su mayor análisis y difusión. Así, varios autores han tratado de modelarlo, primero por medio de modelos lineales y luego por otros no-lineales ${ }^{11}$.

Por su parte Tobin también utiliza un modelo de dos precios el del precio de producción actual de los bienes de capital y el de aquellos ya pre-existentes. Parte de que la riqueza se distribuye entre dos tipos de activos: dinero y capital productivo, afectados por el retorno real del capital productivo y la inflación que sumados constituyen el interés nominal y conforman el costo de oportunidad de mantener efectivo. En su modelo el crecimiento económico se acelera cuando el retorno al dinero disminuye y viceversa. Plantea que una empresa repondrá o adquirirá nuevo capital si considera que éstas le devengarán mayores ganancias y por ende impulsarán el valor de las acciones de la empresa. Finalmente, establece un coeficiente ' $q$ ' definido como la relación del incremento de las ganancias de la empresa valor de mercado de las firmas frente al costo de reemplazo del capital. Si ' $q$ ' es mayor a la unidad, será conveniente reponer el capital, por lo que la inversión crecerá.. Si por el contrario es menor a la unidad será más fácil comprar otras firmas que realizar nuevas inversiones. Así, se tiene un modelo determinístico de equilibrio donde los agentes presentan restricciones presupuestarias.

El coeficiente ' $q$ ' de Tobin se asemeja bastante a la relación establecida por Minsky en la que la inversión tomaría lugar cuando la tasa de retorno esperada por la misma fuese mayor al interés a pagar por la deuda contraída, pero Minsky introduce el riesgo

"Entre los primeros, están los trabajos de Taylor y O'Connell (1985) y el de Lavoie (1986), entre Ios no-lineales destaca el de Peter Skott (1994) y ciertas mediciones realizadas por Dreizzen (1985) 
incurrido tanto por prestamistas como prestatarios en su determinación, pasando el sistema financiero a ser factor determinante en su modelo (Nell, 1994:314).

Esta preponderancia del sistema financiero se evidencia al ser hoy día lugar común hablar del sobredimensionamiento financiero, resultado del inmenso valor de las transacciones realizadas en la esfera financiera en comparación con aquellas de la esfera real, por lo que se habla de hegemonía de lo financiero por sobre lo real. Pero esta hegemonía no es únicamente una característica estática, en tanto que comparación de guarismos, sino que además las variables financieras inducen la inversión real o reproductiva. El sobredimensionamiento financiero, su tasa de crecimiento y la velocidad de movimiento de los flujos de capital, genera presiones que profundizan más esta tendencia, y obliga a revisar desde nuevas perspectivas teóricas el comportamiento de la inversión.

Mata (1999) destaca la existencia constante de una referencia financiera sobre el 'estado de los negocios', obtenida mediante el contraste entre los rendimientos esperados de la inversión reproductiva, las derivadas de la inversión en títulos-valores, la inversión en el mercado cambiario y la tasa de interés (junto con sus correspondientes riesgos asociados). De esta manera, se produce un cálculo ex ante de carácter financiero, o preajuste financiero, que influye en las decisiones de inversión real y por ende en la producción, el empleo y los precios. Por tanto, el equilibrio dinámico general ex post sería el resultado del equilibrio ex ante alcanzado en el mercado de títulos al predeterminarse la inversión para alcanzar determinados niveles de rentabilidad. Se destaca así, una hipótesis central postkeynesiana que es que la velocidad de ajuste del mercado de bienes y servicios es lenta, dada la viscosidad de los precios; y aún más lenta en el mercado laboral, dada la existencia de contratos laborales. A diferencia de lo que sucede en los mercados reales, los mercados de activos financieros (títulos y monedas) se ajustan instantáneamente a las condiciones de costo (tasa de interés) y rendimiento. En este sentido, la variable clave de la política económica pasa a ser la tasa de interés la cual incidirá en el tipo de cambio y finalmente en los precios. O de otra forma, la tasa de interés puede ser vista como el elemento que permite el arbitraje entre todo tipo de inversión, sea ésta en el mercado real, financiero, nacional o internacional. 


\section{Un Modelo}

Las fuentes de financiamiento para la inversión son básicamente dos: las internas o propias de las empresas y las externas o por intermedio de terceros. El autofinanciamiento será posible en la medida que existan beneficios no distribuidos que la empresa decida invertir en la formación del capital reproductivo. Estos beneficios no distribuidos son el resultado de las ganancias del período anterior, las cuales dependerán básicamente de la relación entre las ventas del bien o servicio, su precio y el nivel de rentabilidad de la empresa; y los costos de producción, de realización del bien o servicio y el nivel de endeudamiento existente ${ }^{12}$.

$A_{t+1}=f\left(U_{t}\right)$
$U_{t}=f\left(V_{t}, P_{t}, u_{t}, e_{P}\right)$

Con:

$A_{t+1}$ : Autofinanciamiento para el período siguiente

$U_{t}$ : Utilidades antes de costos financieros del período $\mathrm{t}$

$V_{t}$ : Ventas del período $\mathrm{t}$

$P_{\mathrm{t}}$ : Precio promedio de venta del período $\mathrm{t}$

$u_{t}$ : Índice de rentabilidad de la inversión del período $t$

$\left(u_{t}=U_{t} / K f_{t}=\right.$ utilidad/capital fijo) capital)

$e_{t}$ : Índice de endeudamiento del período $\mathrm{t}\left(e_{t}=L_{t} / K_{t}=\right.$ pasivo $/$

Las fuentes externas para la inversión reproductiva están asociadas al sistema financiero y en este sentido pueden ser de dos tipos: de crédito o de inversión. En el primer caso, la contratación de un crédito dependerá de la relación entre la tasa activa cobrada por las entidades bancarias y la ganancia esperada a partir de la inversión realizada. El pago o servicio de este crédito tendrá dos componente básicos, la amortización del capital y los intereses acumulados a final de cada período. Tomando en cuenta que el sistema

12 Un nivel más detallado de factores obligaría incorporar otros elementos a nivel microeconómico como la depreciación y la tasa impositiva; y a nivel macroeconómico variables como la inflación y la estabiiidad cambiaria, entre otros. 
financiero trabaja a partir del esquema de interés compuesto, el servicio, $\mathrm{S}$, a pagar por un crédito, Cr, sería ${ }^{13}$ :

$$
S=\frac{C r \cdot r}{1-(1+r)^{-n}}
$$

con:

$r$ : tasa de interés activa bancaria

$n$ : número de períodos por los que fue contratado el crédito

En el caso de financiamiento externo por inversión, será el mercado de capitales quien aporte el financiamiento, sea a partir de la emisión de títulos de renta fija o de renta variable. En el caso de la emisión de títulos de renta fija, la empresa contrae un pasivo a una tasa de interés dada, por lo que el resultado para la empresa puede verse similar a la contratación de un crédito. En el caso de la emisión de títulos de renta variable, el financiamiento tendrá como contrapartida una cuota de los beneficios, o visto de otra forma, lo que la empresa ha adquirido es un nuevo socio. En tanto que los beneficios a recibir son inciertos, la evaluación que la empresa realiza sobre la factibilidad y conveniencia de la emisión de títulos de renta variable tendrán como referente los costos ciertos del mercado bancario. De ahí que el financiamiento externo tenga siempre como referente primario a la tasa activa bancaria (preajuste financiero).

A nivel general, la oferta de capital dependerá de la tasa de interés y el riesgo asociado a la inversión y la demanda de capital dependerá de la tasa de rentabilidad esperada y su relación con la tasa de interés.

Partiendo de la taxonomía realizada por Minsky de las empresas en función de su capacidad de cubrir sus compromisos contractuales en unidades Cubiertas, Especulativas y Ponzi, será el tipo de empresa, o su proporción dentro del sector empresarial en su conjunto asociado a las expectativas que acompañan al ciclo económico- lo que permita evidenciar el nivel de fragilidad, el cual resulta inherente al propio proceso de acumulación y más específicamente al proceso de financiamiento de la inversión.

\footnotetext{
${ }^{13}$ Se consideró en la formulación que las anualidades o servicio de la deuda (amortización e intereses) es cancelado al final de cada uno de $\operatorname{los} n$ periodos por los cuales la deuda fue contratada.
} 
Así, ante expectativas favorables, las empresas se embarcan en la adquisición de créditos para la realización de una inversión reproductiva. Por su parte el sistema financiero responde a estas exigencias de crédito generando nuevas fuentes de liquidez. En una primera fase, las empresas muestran flujos positivos y alentadoras expectativas de ganancias, lo cual se convierte en garantía para un mayor financiamiento y se incrementa el nivel de deuda de la empresa. En la medida que la inversión se realiza, la demanda de fondos se hace inelástica a la tasa de interés, la cual tiende a incrementarse. Se observa entonces que el incremento de la fragilidad de la economía es consecuencia natural de un auge inicial de la inversión con intermediación financiera y que puede conducir finalmente a la crisis.

De lo anterior se tiene que el nivel de fragilidad de la empresa dependerá de la relación existente entre las fuentes externas e internas para el financiamiento de la inversión, es decir, entre los compromisos contractuales o servicio de deuda acumulada para el período dado, $\mathrm{S}_{\mathrm{t}}$, y de los niveles de utilidad esperados excluidos los costos financieros, $E\left(U_{t}\right)$. De tal manera que el nivel de fragilidad, $F_{t}$, en un momento dado se podría evaluar a partir de:

$$
\begin{aligned}
& F_{t}=\frac{S_{t}}{E\left(U_{t}\right)} \\
& F_{t}=\frac{\frac{C r \cdot r}{1-(1+r)^{-n}}}{E\left(U_{t}\right)}
\end{aligned}
$$

Se observa entonces como las expectativas futuras afectan el nivel de inversión y grado de endeudamiento deseado por las empresas.

Considerando que el comportamiento de las empresas está claramente vinculado a la fase del ciclo en la cual la economía se halla inmersa, y extrapolando el desenvolvimiento empresarial al ámbito macroeconómico, el nivel de fragilidad de una economía abierta frente a terceros podría ser calculada a partir de:

$$
\begin{aligned}
F_{t} & =\frac{S_{t}}{E\left(B_{t}\right)} \\
B_{t} & =f(\stackrel{+}{X}, \bar{M}, \stackrel{+}{u}, \bar{e})
\end{aligned}
$$




$$
F_{t}=\frac{\frac{D_{t} \cdot r}{1-(1+r)^{-n}}}{E\left(B_{t}\right)}
$$

con:

$B_{t}$ : Balanza Comercial del período $\mathrm{t}$

$X_{t}$ : Exportaciones Totales del período $\mathrm{t}$

$M_{t}$ : Importaciones Totales del período $\mathrm{t}$

$u_{t}$ : índice de inversión de las importaciones del período $t\left(u_{t}=\right.$ $M_{t} / M K f_{t}=$ importaciones totales / importaciones de capital fijo)

$e_{t}$ : Indice de endeudamiento del país en el periodo $t\left(e_{t}=D_{t} /\right.$ $R I N_{t}=$ Deuda Nacional / Reservas Internacionales Netas)

$D_{t}$ : Nivel de deuda acumulada para el período $t$

De tal manera que las expectativas de una economía sobre el comportamiento del mercado financiero internacional, resumido en el comportamiento de la tasa de interés activa bancaria y su relación con las exportaciones que el país es capaz de generar, marcan su capacidad de endeudamiento (financiamiento externo para la inversión reproductiva nacional) y finalmente su nivel de fragilidad.

Una visión dinámica del proceso de fragilización de la economía y su tendencia a la inestabilidad obliga a revisar no sólo los compromisos en determinado momento, sino que exige evidenciar la evolución que el nivel de endeudamiento presenta, según la explicación desarrollada con anterioridad. Así, la relación dinámica determinante a evaluar será la del nivel de deuda acumulada y la capacidad para la generación de fondos, a saber:

$$
\varphi_{t}=D_{t} / B_{t}
$$

con:

$D_{t}$ : Nivel de deuda acumulada al período $t$.

$B_{t}$ : Balanza Comercial del período $\mathrm{t}$

Lo anterior nos permite hacer un análisis del fluctuante proceso de acumulación de la economía, que como se dijese con antelación, está estrechamente imbricado con la generación de nuevas inversiones, las cuales a su vez dependerán de las expectativas sobre el comportamiento del mercado, evaluadas de forma ex-ante a partir del comportamiento de la tasa de interés activa bancaria. De donde: 


$$
I_{t}=k * \Delta X_{t}
$$

con:

$I_{t}$ : inversión realizada en el periodo $t$

$k$ : relación marginal capital-producto

$X_{t}$ : Exportaciones Netas del período $\mathrm{t}$ ( con $\Delta X_{t}=X_{t+1}-X_{t}$ )

Así, el excedente financiero nacional, $E_{t}$, será el resultado del saldo comercial deducidos los pagos financieros por concepto de deuda y la inversión reproductiva realizada en el período:

$$
E_{t}=B_{t}-r \cdot D_{t-1}-I_{t}
$$

De tal manera que el nivel de deuda en un período dado dependerá de la deuda heredada y el excedente generado:

$$
D_{t}=D_{t-1}-B_{t}+r * D_{t-1}+I_{t}
$$

Como el nivel de deuda adquiere significación con relación a la capacidad de generar ingresos, resulta prudente dividir ambos miembros de la ecuación (4) por las exportaciones del período, de donde:

$$
\frac{D_{t}}{X_{t}}=(1+r) \frac{D_{t-1}}{X_{t}}-\frac{B_{t}}{X_{t}}+\frac{I_{t}}{X_{t}}
$$

Tomando en cuenta que:

a) $(1+r) \frac{D_{t-1}}{X_{t}}=(1+r) \cdot \frac{D_{t-1}}{B_{t-1}} \cdot \frac{B_{t-1}}{X_{t-1}} \cdot \frac{X_{t-1}}{X_{t}}$

con:

$$
\begin{aligned}
& \frac{D_{t-1}}{B_{t-1}}=\varphi_{t-1} \\
& \frac{B_{t-1}}{X_{t-1}}=\alpha, \text { relación que indica la capacidad de generación de }
\end{aligned}
$$

fondos (relación de la balanza comercial sobre las exportaciones), coeficiente que se considerará constante en el corto plazo $\left(\frac{B_{t-1}}{X_{t-1}}=\frac{B_{t}}{X_{t}}=\alpha\right)$

$\frac{X_{t-1}}{X_{t}}=\frac{1}{1+g}$, siendo g la tasa de crecimiento de las exportaciones $\left(g=\Delta X_{1} / X_{t}\right)$. 
b) $I_{t} / X_{t}=k g$

Se tiene que:

$\frac{D_{t}}{X_{T}}=\varphi_{t-1} \cdot \alpha\left(\frac{1+r}{1+g}\right)-\alpha+k g$

Asimismo, como:

$$
\begin{aligned}
& \varphi_{t}=\frac{D_{t} / X_{t}}{\alpha} \\
& \varphi_{t}=\varphi_{t-1}\left(\frac{1+r}{1+g}\right)+\frac{k g}{\alpha}-1
\end{aligned}
$$

La resolución de la ecuación en diferencia (7) lleva a:

$$
\begin{aligned}
& \varphi_{t}=\varphi_{t-1} \Rightarrow \varphi_{E}=\left(\frac{1+g}{g-r}\right) *\left(\frac{k g}{\alpha}-1\right) \\
& \varphi_{E}=\varphi_{O}+t\left(\frac{k g}{\alpha}-1\right) \\
& \varphi_{t}=\left(\varphi_{O}-\varphi_{E}\right) \cdot\left(\frac{1+r}{1+g}\right)^{t}+\varphi_{E}
\end{aligned}
$$

Se observa entonces que la dinámica hacia la inestabilidad depende de la relación de la tasa de interés con la del crecimiento de

\begin{tabular}{|c|c|c|c|}
\hline & $K g>a$ & $K g=\alpha$ & $K g<\alpha$ \\
\hline$g>\mathbf{r}$ & $\begin{array}{c}\varphi_{t} \text { converge } \\
\varphi_{E}>\mathbf{0} \\
\text { convergencia rápida }\end{array}$ & $\begin{array}{c}\varphi_{t} \text { converge } \\
\varphi_{E} \rightarrow \mathbf{0}\end{array}$ & $\begin{array}{c}\varphi_{t} \quad \text { converge } \\
\varphi_{E}<\mathbf{0} \\
\text { convergencia lenta }\end{array}$ \\
\hline $\mathbf{g}=\mathbf{r}$ & $\begin{array}{c}\varphi_{t} \text { constante } \\
\varphi_{E}>\varphi_{O}\end{array}$ & $\begin{array}{c}\varphi_{t} \text { constante } \\
\varphi_{E}=\varphi_{O}\end{array}$ & $\begin{array}{c}\varphi_{t} \text { constante } \\
\varphi_{E}<\varphi_{O}\end{array}$ \\
\hline $\mathbf{g}<\mathbf{r}$ & $\begin{array}{c}\varphi_{t} \text { diverge } \\
\varphi_{E}>\mathbf{0} \\
\text { divergencia lenta }\end{array}$ & $\begin{array}{l}\varphi_{,} \text {diverge } \\
\varphi_{E} \rightarrow \infty\end{array}$ & $\begin{array}{c}\varphi_{t} \quad \text { diverge } \\
\varphi_{E}<0 \\
\text { divergencia rápida }\end{array}$ \\
\hline
\end{tabular}
las exportaciones, de forma tal que el acervo de deuda mantiene una relación directa con la primera e inversa frente a la segunda y su velocidad de convergencia o divergencia dependerá del valor asumido por $\mathrm{kg} / \mathrm{a}$ : 
Considerando como premisa la perspectiva postkeynesiana de ajustes diferenciales en el tiempo de los distintos mercados, la tasa de interés será la de más rápido ajuste, lo que conlleva a un preajuste financiero de la inversión para la exportación. El impacto de la tasa de crecimiento de las exportaciones en la dinámica del acervo de deuda, o su tasa instantánea de variación, se resume entonces en:

$$
\partial \varphi_{t} / \partial g=-\varphi_{t-1} \cdot \frac{(1+r)}{(1+g)^{2}}+\frac{k}{\alpha}
$$

y su incidencia es de dos formas: por un lado y en sentido inverso, un incremento de las exportaciones permite la generación de fondos para la reducción de la deuda y por otro lado, un incremento de las exportaciones exige una mayor inversión para la generación de las misma y por tanto, favorece un aumento de la deuda.

\subsection{Tasas Fluctuantes Cíclicas}

En el acápite anterior se ha considerado que la tasa de interés y la de crecimiento de las exportaciones son constantes, de ahí que el comportamiento o dinámica de la deuda sea finalmente de carácter lineal. Pero el comportamiento de las variables macroeconómicas presenta, en teoría, una dinámica fluctuante o ajustada a la fase del ciclo en la cual se halla inmersa la economía. Si la tasa de interés tomada en cuenta es la real, $r$, la misma podrá ser considerada constante, ya que la tasa de interés nominal, $i$, se ajusta por la tasa inflacionaria, $\pi$, de forma que:

$$
(1+i)=(1+r) *(1+\pi)
$$

Pero la tasa de crecimiento de las exportaciones será fluctuante y su comportamiento será dependiente de la etapa del ciclo en la cual se encuentre el mercado internacional, receptor de las exportaciones; y podrá ser descrita a partir de:

$$
g=\lambda \operatorname{Sen}(t)
$$

siendo el Rango de $g=[-\lambda, \lambda]$

A partir de esta formulación de la tasa de crecimiento de las exportaciones, logramos que la misma establezca ciclos constantes de amplitud $t$ y longitud dependiente de $\lambda$. Se reconoce que los ciclos económicos no muestran en la realidad tal nivel de precisión en la fluctuación, pero el uso de la función trigonométrica en el in- 
dicador dinámico de inestabilidad, permitirá que el comportamiento derivado sea más ajustado y por ende más útil para el análisis de $\varphi_{t}$. De tal forma que al incorporarla a (7) resulta:

$$
\varphi_{t}=\varphi_{t-1}\left(\frac{1+r}{1+\lambda \operatorname{Sen}(t)}\right)+\frac{k \cdot \lambda \operatorname{Sen}(t)}{\alpha}-1
$$

y cuya solución es:

$$
\begin{aligned}
& \varphi_{t}=\left(\varphi_{O}-\varphi_{E}\right) *\left(\frac{1+r}{1+\lambda \operatorname{Sen}(t)}\right)^{t}+\varphi_{E} \\
& \varphi_{E}=\left(\frac{1+\lambda \operatorname{Sen}(t)}{\lambda \operatorname{Sen}(t)-r}\right) *\left(\frac{k \cdot \lambda \operatorname{Sen}(t)}{\alpha}-1\right) \\
& \varphi_{E}=\varphi_{O}+t\left(\frac{k \cdot \lambda \operatorname{Sen}(t)}{\alpha}-1\right)
\end{aligned}
$$

La Gráfica \#1 muestra en primer lugar una serie de iteracioñes de (11) para determinados valores de $\lambda$ y diferentes estructuras dinámicas a lo largo de su evolución en el tiempo.

Se observa que $\varphi_{t}$ no está definida cuando $(1+\lambda \operatorname{Sen}(t))=0$, es decir para $t=\operatorname{arcSen}(-1 / \lambda) \quad$ ó $n=t / \pi=\frac{\operatorname{arcSen}(-1 / \lambda)}{\pi}+2 k \pi$.

De lo anterior, destaca la importancia de lo prónunciado del ciclo $(\lambda)$, evidenciado por la tasa de crecimiento de las exportaciones, para la continuidad en la dinámica del indicador. En esta oportunidad, al evaluar la dinámica de $\varphi_{t}$, se tiene que:

$$
\varphi_{t}^{\prime}=\frac{1+r}{1+\lambda \operatorname{Sen}(t)}=f(t)
$$

El punto crítico evidenciado en la ecuación anterior está en $\mathrm{r}=\bullet 1$, nuevamente la función no existe para $t=\operatorname{arcSen}(-1 / \lambda)$ ó $n=t / \pi=\frac{\operatorname{arcSen}(-1 / \lambda)}{\pi}+2 k \pi$ y la dinámica se indetermina al coincidir las dos situaciones previamente indicadas. El comportamiento de (13) se observa en las gráficas \#2, \#3 y \#4 para diferentes valores de $\lambda$. En la medida que $\lambda$ crece, es decir que los ciclos de la tasa de crecimiento son más pronunciados, la fluctuación evidenciada se 
hace a su vez más frecuente, dando como resultado una mayor inestabilidad en el comportamiento del indicador.

Se observa así, que la tasa de variación del indicador de inestabilidad dinámica es de por sí fluctuante y por tanto su propia dinámica con respecto al tiempo es evidenciada por:

$$
\frac{d f(t)}{d t}=\frac{-\lambda \operatorname{Cos}(t) \cdot(1+r)}{(1+\lambda \operatorname{Sen}(t))^{2}}=\frac{-\lambda \operatorname{Cos}(t) \cdot(1+r)}{1+\lambda^{2} \operatorname{Sen}^{2}(t)+2 \lambda \operatorname{Sen}(t)}
$$

En este caso, al igual que en los anteriores la función no existe en la medida que $(1+\lambda \operatorname{Sen}(t))=0$, es decir para $t=\operatorname{arcSen}(-1 / \lambda)$ ó $n=t / \pi=\frac{\operatorname{arcSen}(-1 / \lambda)}{\pi}+2 k \pi ; \mathrm{y}$ evidencia puntos críticos para $\mathrm{r}=-1$ y para $\mathrm{t}=\pi / 2+2 \mathrm{k} \pi$ ó $\mathrm{n}=\mathrm{I} / 2$, de forma tal que la función se indetermina cuando $\mathrm{r}=-1$. y $t=\operatorname{arcSen}(-1 / \lambda)$.

El comportamiento de la variación instantánea de $f(t)$ se aprecia en las gráficas \#5, \#6 y \#7, nuevamente para diversos valores de $\lambda \mathrm{y}$ en ellas se constata la importancia que tiene la profundidad de los ciclos, en el indicador de inestabilidad. Al igual que en las gráficas anteriores, las que muestran valores de $\lambda$ sumamente pequeños, son aquellas con ciclos menos abruptos ${ }^{14}$.

\subsubsection{Tasa de interés nominal $V_{s}$. Tasa de interés real}

Si bien es cierto que la evaluación de la tasa de interés en el largo plazo ha de hacerse a valor real, no lo es menos, que en el corto plazo la relación de pagos contractuales está definida por su valor nominal. De tal manera que si en el análisis se hace uso de la tasa de interés nominal, en vez de la real, no se podrá considerar esta variable como una constante, ya que no estará presente ningún factor que la descuente (índice de precios). La dinámica de la tasa de interés nominal, $i$, en teoría debería ser de igual dirección y sen-

\footnotetext{
14 Vale recordar que los picos (cimas y fosas) evidenciados en las gráficas nos son tales, sino ajustes realizados por los programas que nos han permitido las mismas, en este caso MAPLE $8{ }^{\circledR}$. A cada pico corresponde una zona en la que la función no existe o se presenta indeterminación de la función (valores en el texto).
} 
tido que la de la tasa de crecimiento de las exportaciones, ya que ambas han de manifestar la fase del ciclo en la cual la economía se halla presente. En este caso, podemos hacer similar ajuste a la tasa de interés que el realizado a la del crecimiento de las exportaciones y definirla como:

$$
i=\gamma \operatorname{Sen}(t)
$$

siendo el Rango de $\mathrm{i}=(-\gamma, \gamma)$

En este caso:

$$
\varphi_{t}=\varphi_{t-1}\left(\frac{1+\gamma \operatorname{Sen}(t)}{1+\lambda \operatorname{Sen}(t)}\right)+\frac{k \cdot \lambda \operatorname{Sen}(t)}{\alpha}-1
$$

La solución de la ecuación en diferencia (16), viene dada por:

$$
\begin{aligned}
\varphi_{t} & =\left(\varphi_{O}-\varphi_{E}\right) *\left(\frac{1+\gamma \operatorname{Sen}(t)}{1+\lambda \operatorname{Sen}(t)}\right)^{t}+\varphi_{E} \\
\varphi_{E} & =\left(\frac{1+\lambda \operatorname{Sen}(t)}{(\lambda-\gamma) \operatorname{Sen}(t)}\right) \cdot\left(1-\frac{k \cdot \lambda \operatorname{Sen}(t)}{\alpha}\right) \\
\varphi_{E} & =\varphi_{O}+t\left(\frac{k \cdot \lambda \operatorname{Sen}(t)}{\alpha}-1\right)
\end{aligned}
$$

La dinámica de la inestabilidad depende a su vez de:

$$
\varphi_{t}^{\prime}=\left(\frac{1+\gamma \operatorname{Sen}(t)}{1+\lambda \operatorname{Sen}(t)}\right)=f(t)
$$

De igual manera que en el caso de se utilizara la tasa de interés real (considerada constante), al utilizar la tasa de interés nominal, la función deja de existir en la medida que $(1+\lambda \operatorname{Sen}(t))=0$, es decir para $t=\operatorname{arcSen}(-1 / \lambda)$ ó $n=t / \pi=\frac{\operatorname{arcSen}(-1 / \lambda)}{\pi}+2 k \pi$.

En esta oportunidad la dinámica del indicador muestra un punto crítico para: $1+\gamma \operatorname{Sen}(t)=0$, es decir para $t=\operatorname{arcSen}(-1 / \gamma)$ ó $n=t / \pi=\frac{\operatorname{arcSen}(-1 / \gamma)}{\pi}+2 k \pi$. El comportamiento de la dinámica del indicador se observa en las series gráficas \#8 y \#9. Estas series revelan la importancia de $\lambda$ y $\gamma$ en la estructura de la dinámica y en los niveles alcanzados por el indicador dinámico. 
La variación instantánea en el tiempo de $\mathrm{f}(\mathrm{t})$ vendrá dado por:

$$
\frac{d f(t)}{d t}=\frac{(\gamma-\lambda) \cdot \operatorname{Cos}(t)}{(1+\lambda \operatorname{Sen}(t))^{2}}
$$

presentando puntos críticos cuando $t=\pi / 2+2 k \pi$ ó $n=1 / 2$, o cuando $\lambda=\gamma$. Al igual que en casos anteriores la función deja de existir cuando $t=\operatorname{arcSen}(-1 / \lambda)$ ó $n=t / \pi=\frac{\operatorname{arcSen}(-1 / \lambda)}{\pi}+2 k \pi$. El comportamiento de dicha variación instantánea se observa en las series gráficas \#10 y \#11 y al igual que en el comportamiento de la dinámica del indicador, se revela la importancia de $\lambda$ y $\gamma$.

\subsubsection{Ciclos distintos: dirección, sentido, longitud y amplitud}

La tasa de interés nominal no necesariamente ha de manifestar un comportamiento acompasado al de la tasa de crecimiento de las exportaciones y por tanto la dinámica reflejada puede ser distinta à la de dicha variable. Esto puede ocurrir por distintas razones:

- La tasa de interés puede ser una variable externa a la economía, cosa que sucede cuando los préstamos adquiridos para la inversión son de capital foráneo y por ende el movimiento reflejado por dicha tasa acusa el comportamiento de una economía distinta a la del receptor del capital.

- Tomando en cuenta los postulados del preajuste financiero, si bien la tasa de interés podría moverse en igual dirección y sentido que la tasa de crecimiento de las exportaciones, al hacerlo a una velocidad mayor, su movimiento queda desacompasado al de dicha variable.

- La tasa de interés y la tasa de crecimiento de las exportaciones pueden iniciar su fluctuación simultáneamente pero al hacerlo con ondas de amplitud y longitud distintas se genera desalineamiento de las mismas.

Se podrían representar situaciones de esta índole a partir de modificar una de las funciones de las tasas, en este caso, la de i, a saber:

$$
i=\gamma \operatorname{Cos}(t)
$$


siendo el Rango de $\mathrm{i}=(-\gamma, \quad \gamma)$; y en este caso,

$$
\varphi_{t}=\varphi_{t-1}\left(\frac{1+\gamma \operatorname{Cos}(t)}{1+\lambda \operatorname{Sen}(t)}\right)+\frac{k \cdot \lambda \operatorname{Sen}(t)}{\alpha}-1
$$

La solución de la ecuación en diferencia de (21) viene dada por:

$$
\begin{aligned}
& \varphi_{t}=\left(\varphi_{O}-\varphi_{E}\right) *\left(\frac{1+\gamma \operatorname{Cos}(t)}{1+\lambda \operatorname{Sen}(t)}\right)^{t}+\varphi_{E} \\
& \varphi_{E}=\left(\frac{1+\lambda \operatorname{Sen}(t)}{\lambda \operatorname{Sen}(t)-\gamma \operatorname{Cos}(t)}\right) \cdot\left(1-\frac{k \cdot \lambda \operatorname{Sen}(t)}{\alpha}\right)
\end{aligned}
$$

$\varphi_{E}=\varphi_{O}+t\left(\frac{k \cdot \lambda \operatorname{Sen}(t)}{\alpha}-1\right)$

para $\mathrm{r}=\mathrm{g}$

$\varphi_{t}^{\prime}=\frac{1+\gamma \operatorname{Cos}(t)}{1+\lambda \operatorname{Sen}(t)}=f(t)$

En esta oportunidad la dinámica del indicador muestra un punto crítico para $1+\gamma \operatorname{Cos}(t)=0$, es decir para $t=\arccos (-1 / \gamma)$ ó $n=t / \pi=\frac{\operatorname{arcCos}(-1 / \gamma)}{\pi}+2 k \pi$, dejando de existir la función cuando $1+\gamma \operatorname{Sen}(t)=0, \quad$ es $\quad$ decir, $\quad$ para $t=\operatorname{arcSen}(-1 / \lambda) \quad$ ó $n=t / \pi=\frac{\operatorname{arcSen}(-1 / \lambda)}{\pi}+2 k \pi ;$ y cuyo comportamiento se observa en las series gráficas \#12 y \#13.

La variación instantánea en el tiempo de $f(t)$ vendrá dado por:

$$
\frac{d f(t)}{d t}=\frac{-\gamma \operatorname{Sen}(t)-\lambda \operatorname{Cos}(t)-\gamma \lambda}{(1+\lambda \operatorname{Sen}(t))^{2}}
$$

de donde el punto crítico se manifiesta cuando $\gamma \operatorname{Sen}(t)+\lambda \operatorname{CoS}(t)=-\gamma \cdot \lambda$, y la función no existe para $1+\gamma \operatorname{Sen}(t)=0$, es decir, para $t=\operatorname{arcSen}(-1 / \lambda)$ ó $n=t / \pi=\frac{\operatorname{arcSen}(-1 / \lambda)}{\pi}+2 k \pi$. 
Su comportamiento se observa en las series gráficas \#14 y \#15; y evidencian la importancia de la relación tanto en dirección, sentido como de longitud de las fluctuaciones evidenciadas por la tasa de interés y la de crecimiento de las exportaciones.

\section{Reflexiones Finales}

El modelo desarrollado ha permitido evaluar dos casos básicos a partir del comportamiento de las tasas relevantes para nuestro estudio (tasa interés y tasa de crecimiento de las exportaciones), a saber:

- Interés y crecimiento constante. En este caso, la dinámica hacia la inestabilidad depende de la relación de la tasa de interés con la del crecimiento de las exportaciones, de forma tal que el acervo de deuda mantiene una relación directa con la primera e inversa frente a la segunda y su velocidad de convergencia o divergencia dependerá del valor asumido por el producto de la relación marginal capital-producto y la tasa de crecimiento de las exportaciones frente la capacidad de generar exportaciones en el saldo de balanza comercial $(\mathrm{kg} / a)$.

- Interés y crecimiento fluctuante: En este caso la dinámica hacia la inestabilidad depende de la relación de amplitud, longitud, dirección y sentido de las fluctuaciones evidenciadas por las tasas evaluadas, de forma tal que los valores absolutos de las tasas no explican de suyo la convergencia o divergencia de la tendencia.

Dentro de este caso se evaluaron tres posibilidades: tasa de interés constante / tasa de crecimiento fluctuante, ambas tasas fluctuando de forma acompasada, ambas tasas fluctuando de forma no acompasada. El análisis de la dinámica permite a concluir que su estabilidad / inestabilidad se explica cada vez menos por los valores absolutos de las tasas en la medida que la longitud de sus fluctuaciones se hace más diferenciada.

De lo anterior se infiere una mayor inestabilidad en los casos de ciclos no acompasados que en los acompasados y de estos últimos por sobre los que presentan tasas constantes.

Se ha observado a partir del modelo que movimientos cíclicos de determinadas variables, producidos como consecuencia de las fluctuaciones de la economía, devienen en dinámicas marcadamente irregulares. Las dinámicas resultantes impiden que políticas deriva- 
das de esquemas convencionales, lineales, atemporales y con excesiva simplificación tengan los efectos equilibrantes que buscan; de donde más que una crítica a las políticas, es una impugnación a la aceptación rígida de los marcos teórico-metodológicos de las que emanan y evidencia de la imperiosa necesidad de la incorporación de nuevas y más amplias perspectivas.

Asimismo, resalta que la incorporación en la formulación del comportamiento de las tasas relevantes para el estudio de funciones trigonométricas básicas, calificables como funciones matemáticas 'buenas' (en el sentido de diferenciables indefinidamente), generan inestabilidades importantes en las dinámicas de las variables económicas bajo análisis. Esta situación se puede visualizar al cruzar los cortes realizados a las distintas funciones graficadas, a partir de los ejes de tiempo, el de la onda de la tasa de crecimiento $(\lambda \gamma)$ y el de la onda de la tasa de interés $(\gamma)$. De esta manera podemos imaginar una figura tridimensional donde los cortes generan isosuperficies irregulares, cualquiera que sea el eje utilizado.

El último de los casos planteados, tasas de interés y de crecimiento fluctuantes de forma desacompàsada, tiene particular interés para economías pequeñas / medianas y abiertas, pues el capital requerido para sus inversiones es, en un importante porcentaje, de carácter externo, de ahí que la dinámica de su precio (tasa de interés) no esté acompasada a las necesidades y realidades de la economía prestataria.

La inestabilidad financiera de una economía adquiere gran significación a partir del análisis de indicadores del sector público y del resultado de sus políticas. Desde el punto de vista de las finanzas públicas, la inestabilidad financiera nacional se podría asimilar a una situación donde la política fiscal se hace insostenible, es decir un escenario en el cual los mercados voluntarios financieros no permiten la gestión del déficit del sector público. El desarrollo de indicadores de sostenibilidad fiscal a partir de los aportes teóricos previos, resultarían de interés tanto en el plano teórico como aplicación práctica de dichas contribuciones.

Lo anterior nos lleva a insistir en la importancia de los estudios no lineales y de la inestabilidad en el análisis económico, como fuente de complejidad en la dinámica económica, los cuales no deben ser entendidos como fenómenos puntuales, aleatorios o perturbadores. Más aún, las interacciones dinámicas de diferentes fuerzas no lineales 
en un proceso evolutivo, tienen amplias posibilidades de generar caos endógeno, concepto alejado de los estudios centrados en el equilibrio en la economía tradicional, pero claramente presentes en la dinámica económica actual.

\section{Referencias}

Arrighi, Giovanni (1998) "Globalization and the Rise of East Asia. Lessons form the Past, Prospects for the Future" International Sociology. Vol.13, March (1):59-77.

Arrighi, Giovanni y Beverly Silver.(1999) Chaos and Governance in The Modern World System. Univ. Minnesota Press.

Baumol William J. y Jess Benhabib (1989) "Chaos: Significance, Mechanism and Economic Applications" Journal of Economic Perspectives, Vol.3, Winter (1): 77 105.

Dreizzen, Julio (1985) Fragilidad Financiera e Inflación, Centro de Estudios de Estado y Sociedad, CEDES, Buenos Aires, Argentina.

Dymski, Gary y Robert Pollin (1992) "Hyman Minsky as Hedhog: The Power of the Wall Street Paradigm" en: Fazzari, Steven y Dimitri Papadimitriou (eds.) Financial Conditions and Macroeconomic Performance, ME Sharpe Inc, London/NY, pp2762.

Earley, James (1997) "Joseph Schumpeter: a Frustrated 'Creditist"' en: New Perspectives in Monetary Macroeconomics. Dymski, Gary y Robert Pollin, eds. University Michigan Press.

Fisher, Irving (1907) The Rate of Interest: Its nature, determination and relation to economic phenomena. MacMillan, NY.

Kalecki, M (1965) Theory of Economic Dynamics, Allen and Unwin.

Keynes, John Maynard (1930) The Treatise of Money. Harcourt, Brace and Co., NY. Keynes, John Maynard (1980 [1943]) Teoría General de la Ocupación, el Interés y el Dinero. Fondo de Cultura Económica. Traducción de: Eduardo Hornedo. Título Original: The General Theory of Employment, Interest and Money (1936). Kindleberger, Charles (1996 [1978]) Manias, Panics and Crashes. A History of Financial Crises. 3ra. Ed. John Wiley $\mathcal{Z}$ Sons, Inc.

Kregel Jan (1992) "Minsky's 'two price' theory of financial instability and monetary policy: discounting versus open market intervention" Fazzari, Steven y Dimitri Papadimitriou (eds.) Financial Conditions and Macroeconomic Performance, ME Sharpe Inc, London/NY, pp85-104. 
Lavoie Marc (1985) "Credit and Money: the dynamic circuit, overdraft economics and postkeynesian economics" en: Money and Macro Policy, Jasulic Marc ed,. Kluwer-Nijhoff Pub., Boston: 63-84.

Lavoie, Marc (1986) "Systemic Financial Fragility: a Simplified View" Journal of Post Keynesian Economics, Vol.9, Winter (2): 258-266.

Marx, Carlos (1977 [1867]) El Capital, Tomo I, II, III. Fondo de Cultura Económica, Bogotá. Título Original: Das Kapital. Traducción de Wenceslao Roces.

Mata, Luis (1999) Economía Alternativa, CEAP-Tropykos, Caracas, Venezuela

Minsky, Hyman (1982) Can't it happend again. Essays on Instability and Finance. M.E Sharpe, Inc., Armonk, NY.

Minsky, Hyman (1996) "The Essential Characteristics of PostKeynesian Economics" en Deleplace, Ghislain y Edward Nell (Eds), Money in Motion. MacMillan Press Ltd, London, pp70-88.

Mitchell, Wesley (1913) Business Cycles. Univ. of California Press, Berkeley.

Nasica, Eric (2000) Finance, Investment and Economic Fluctuations, Edward Elgar Pub., UK y EUA.

Nell, Edward (1994) "Minsky, Keynes and Sraffa: Investment and the Long Period en: New Pespectives in Monetary Macroeconomics. Explorations in the Tradition of Hyman P. Minsky. Dymski Gary y Robert Pollin Eds. Ann Arbor Univ. of Michigan Press

Taylor, Lance y Stephen O'Connell (1985) "A Minsky Crisis" Quarterly Journal of Economics, Vol 100 (supplement): 871-885

Wallerstein, Inmanuel (1991) Geopolitics and Geoculture: Essays on the Changing World-System. Cambridge Univ. Press.

Wicksell, Knut (1962 [1936, 1898]) Interest and Prices. Augustus M. Kelley, NY

Wolfson, Martin (1990) "The Causes of Financial Instability" Journal of Postkeynesian Economics, Vol.12, Spring (3): 333-355.

Wray Randall (1992) "Minsky's Financial Instability Hipótesis and the Endogeneity of Money" en: Fazzari, Steven y Dimitri Papadimitriou (eds.) Financial Conditions and Macroeconomic Performance, ME Sharpe Inc, London/NY, pp161-180. 


\section{Anexo}

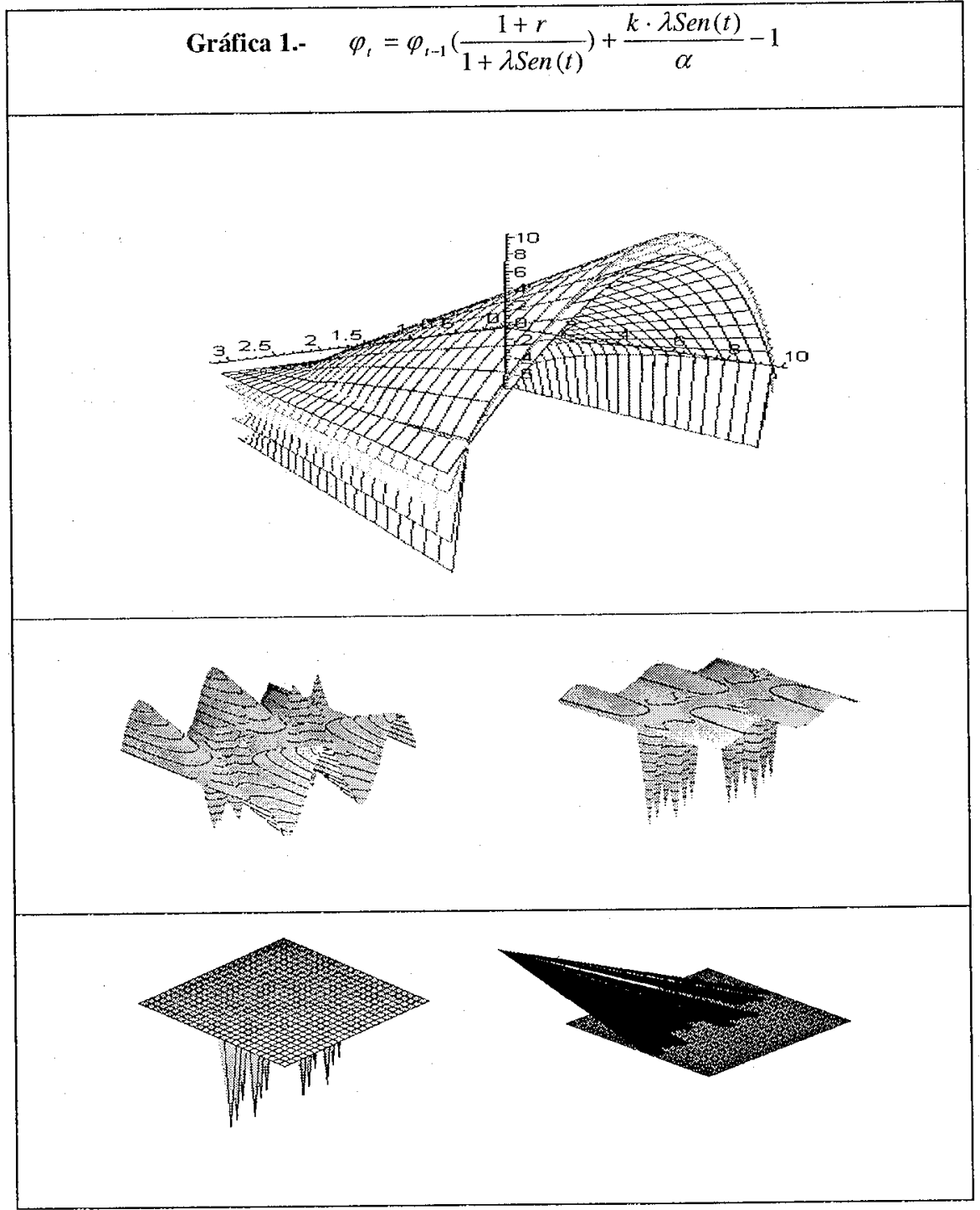




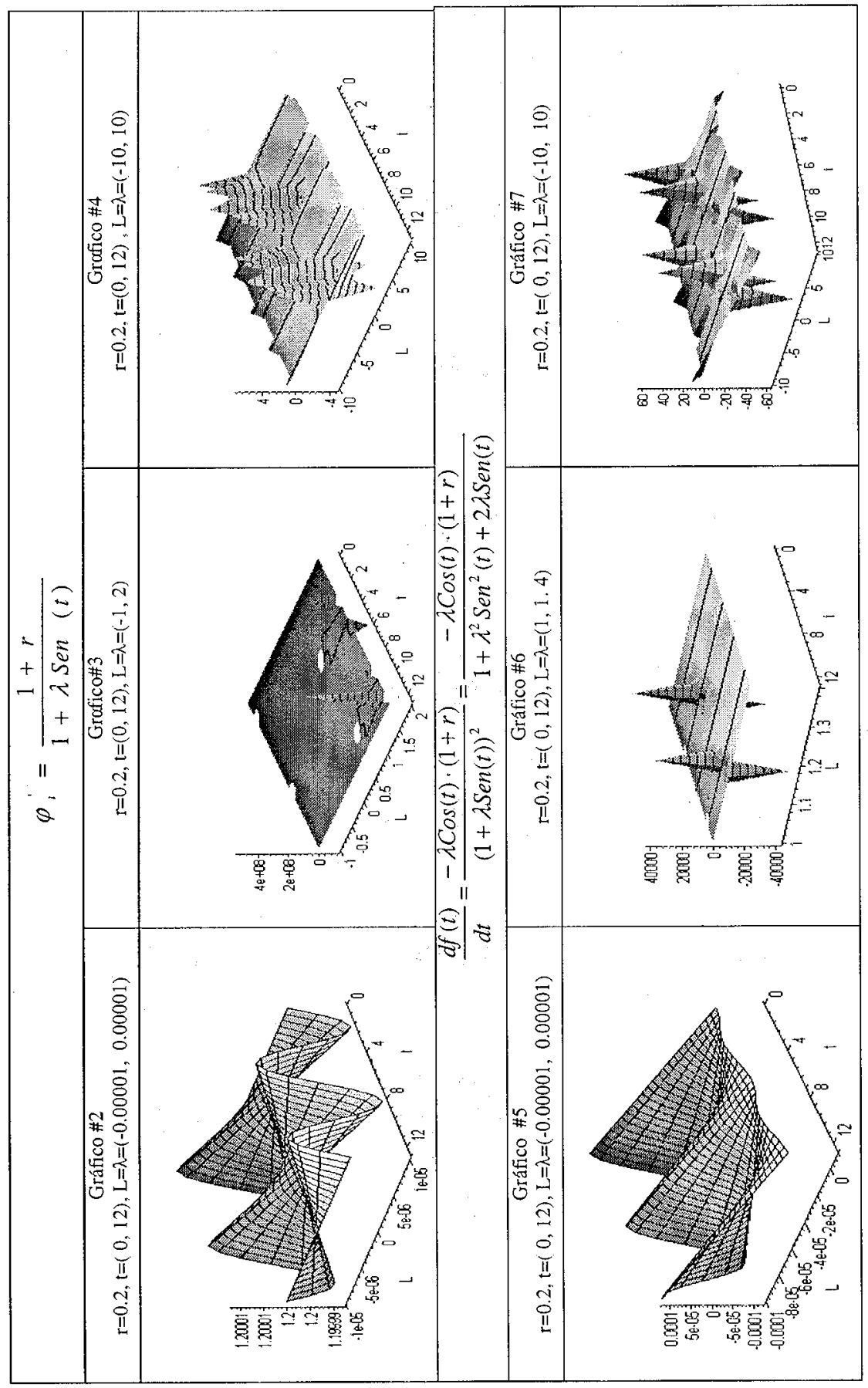

Revista Análise Econômica, ano 22, n. 41 


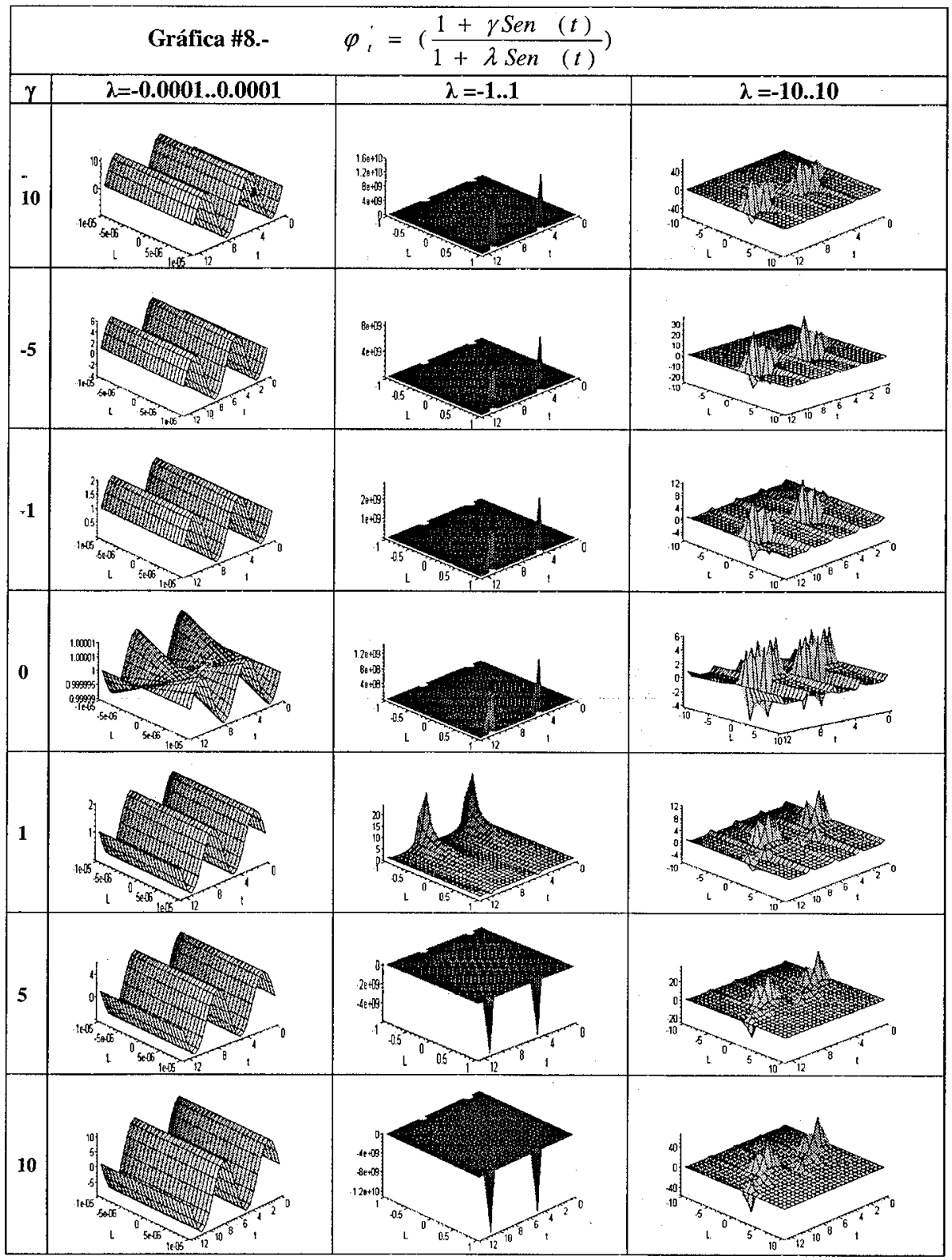




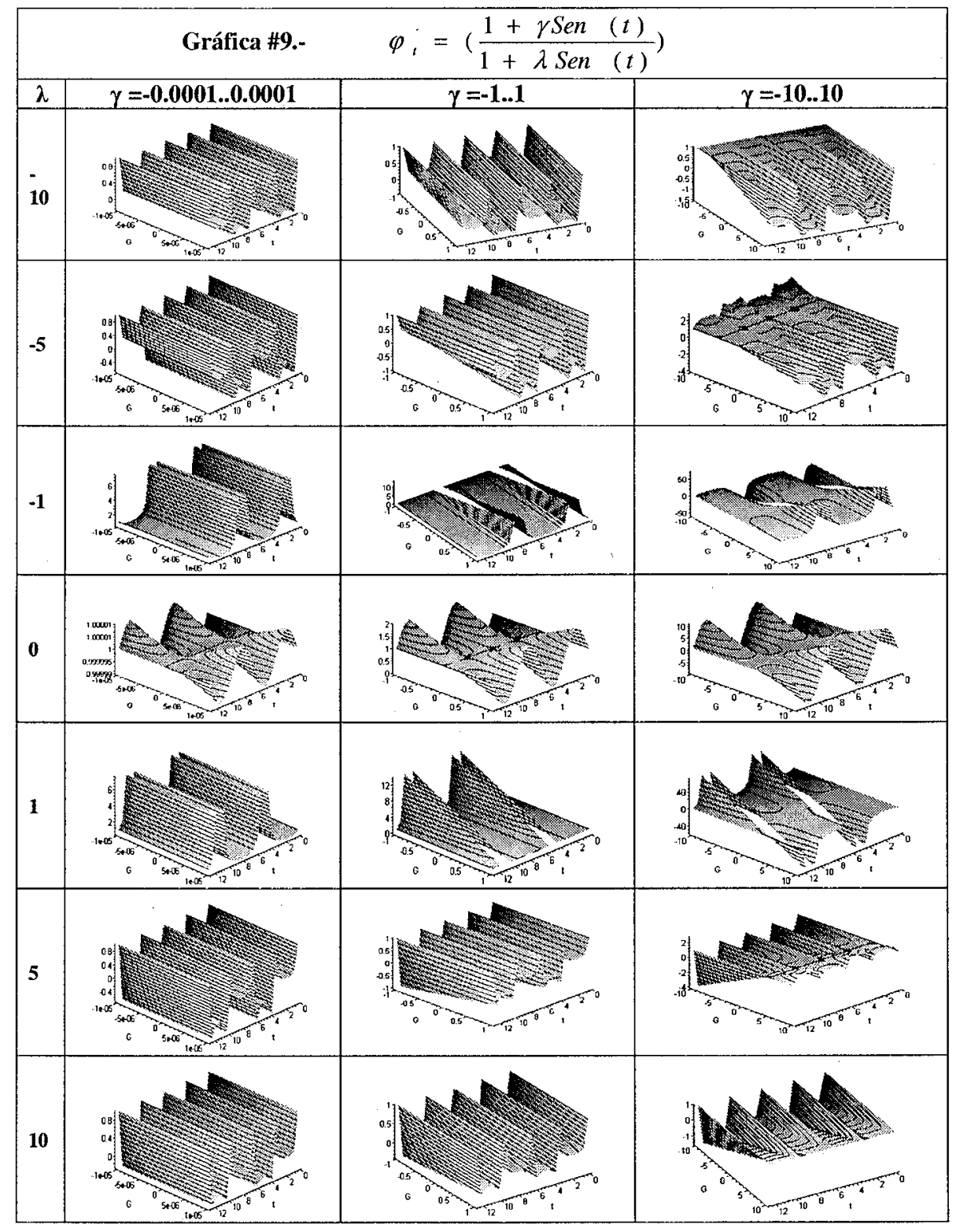




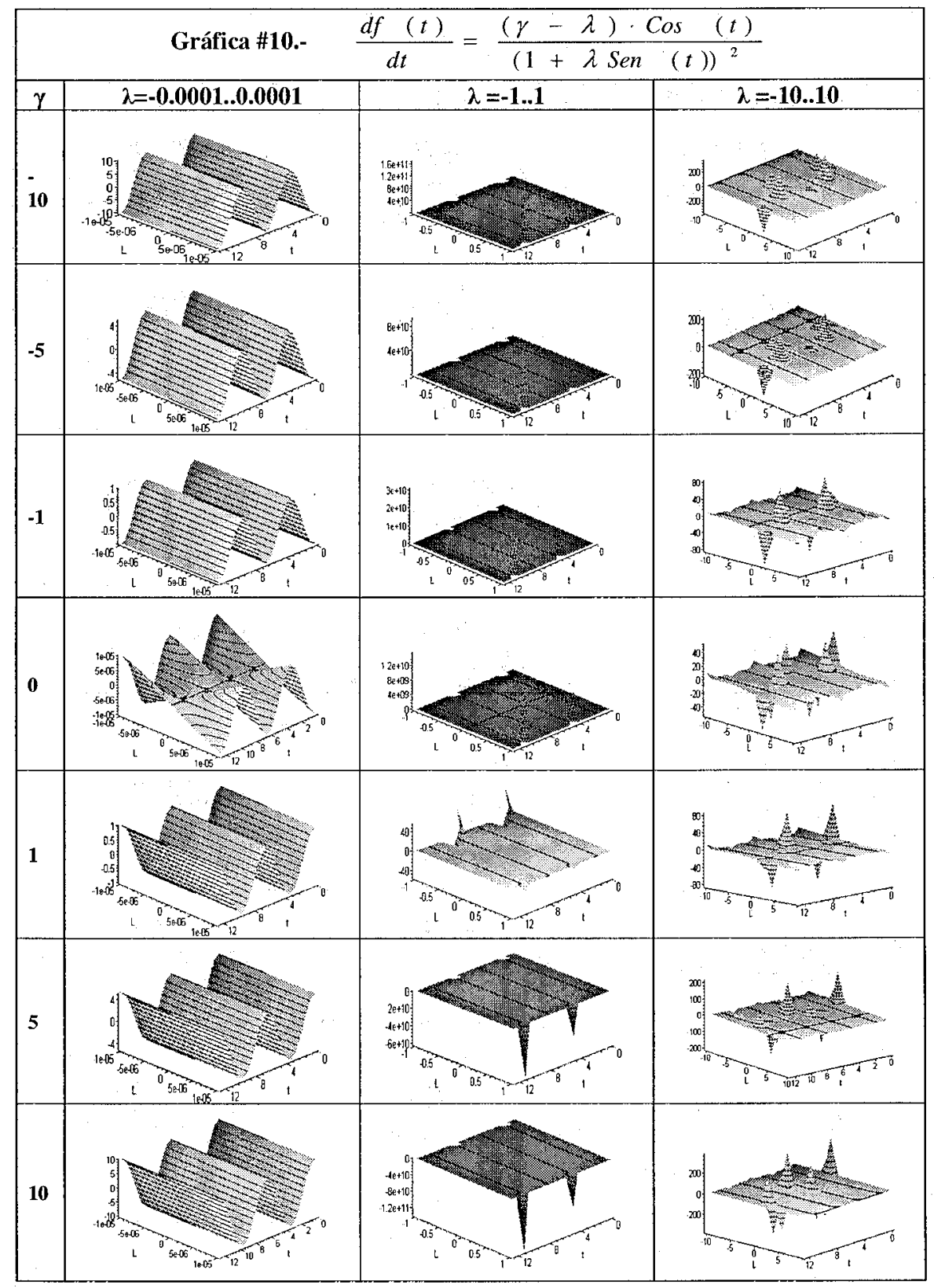




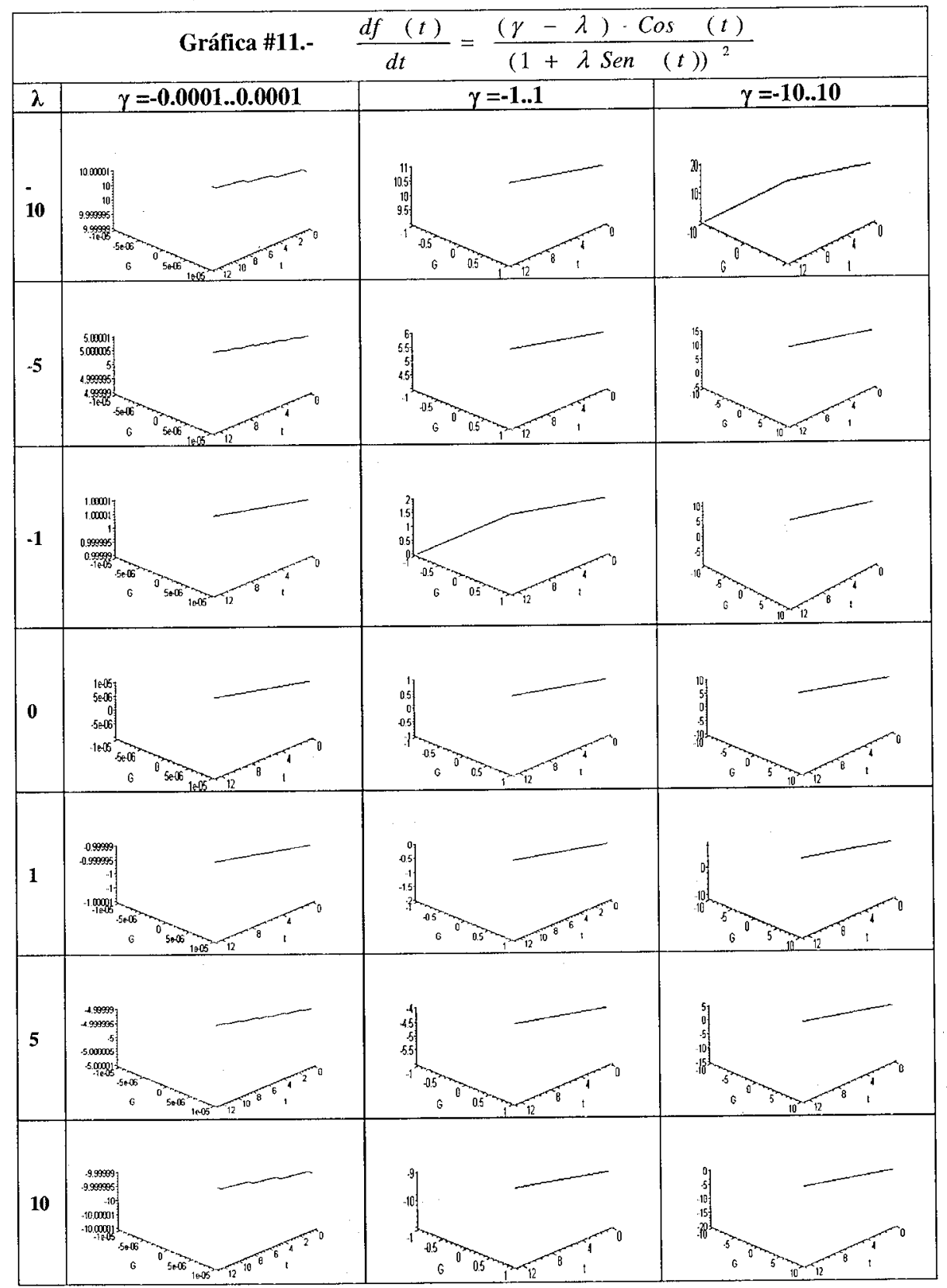




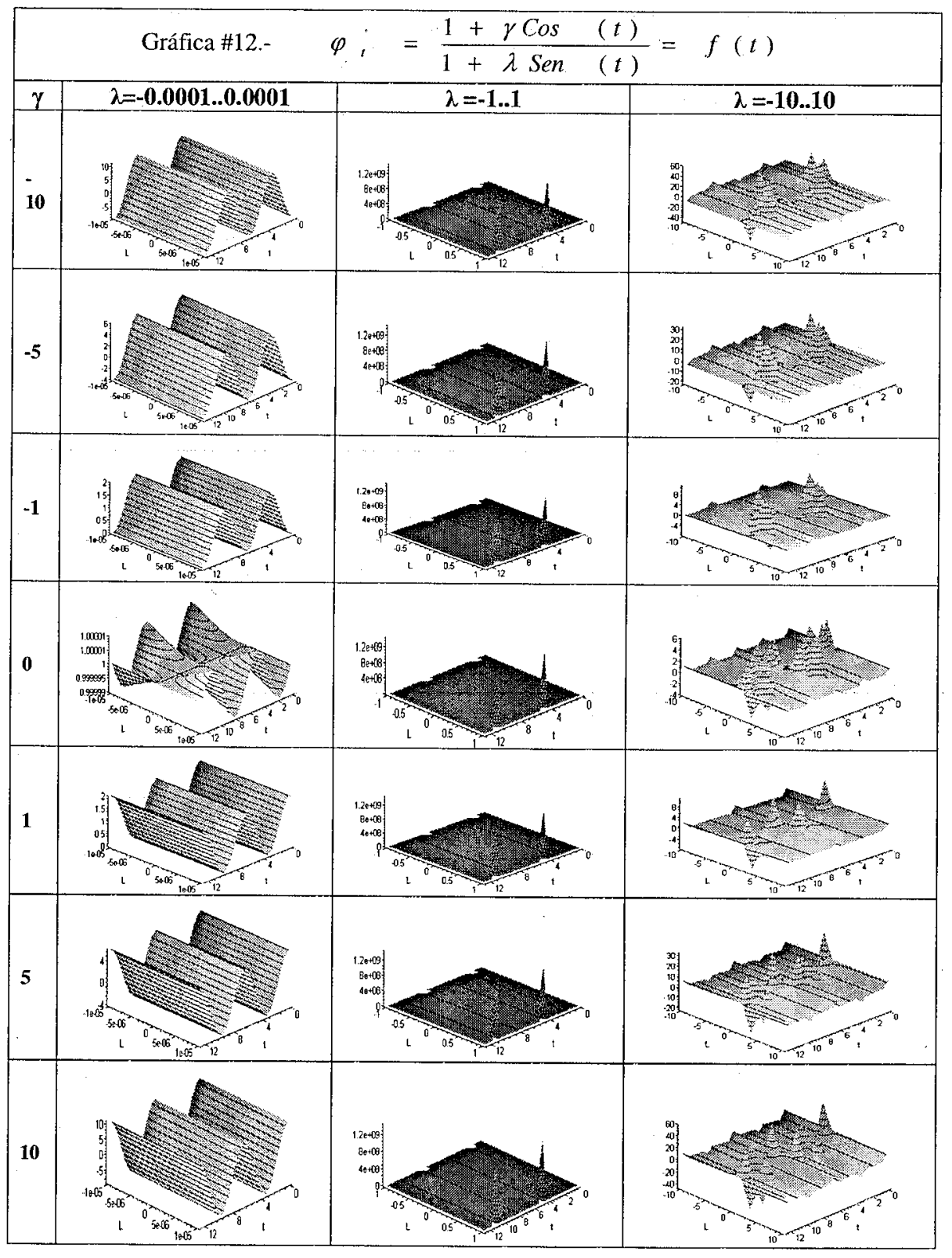




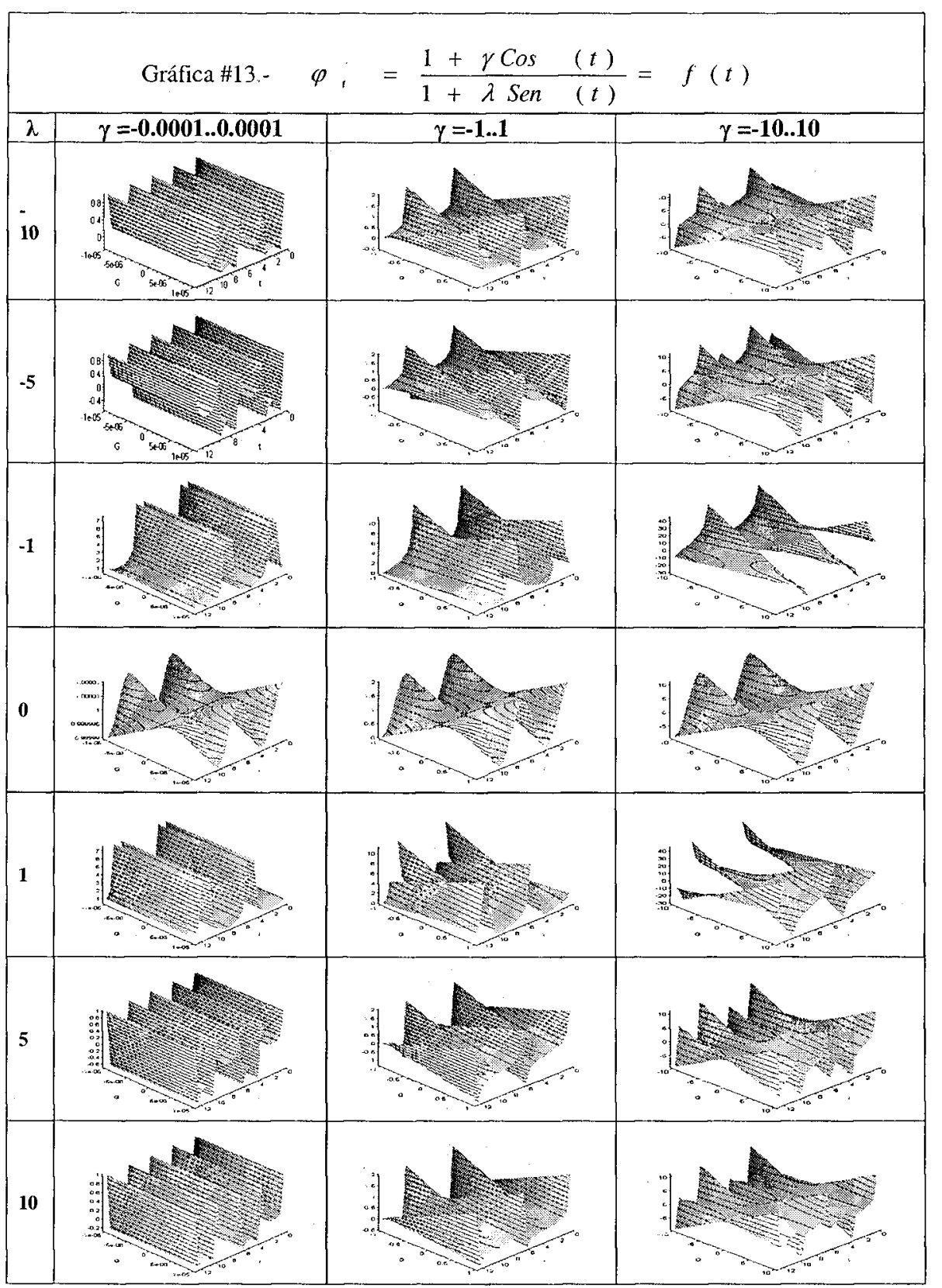




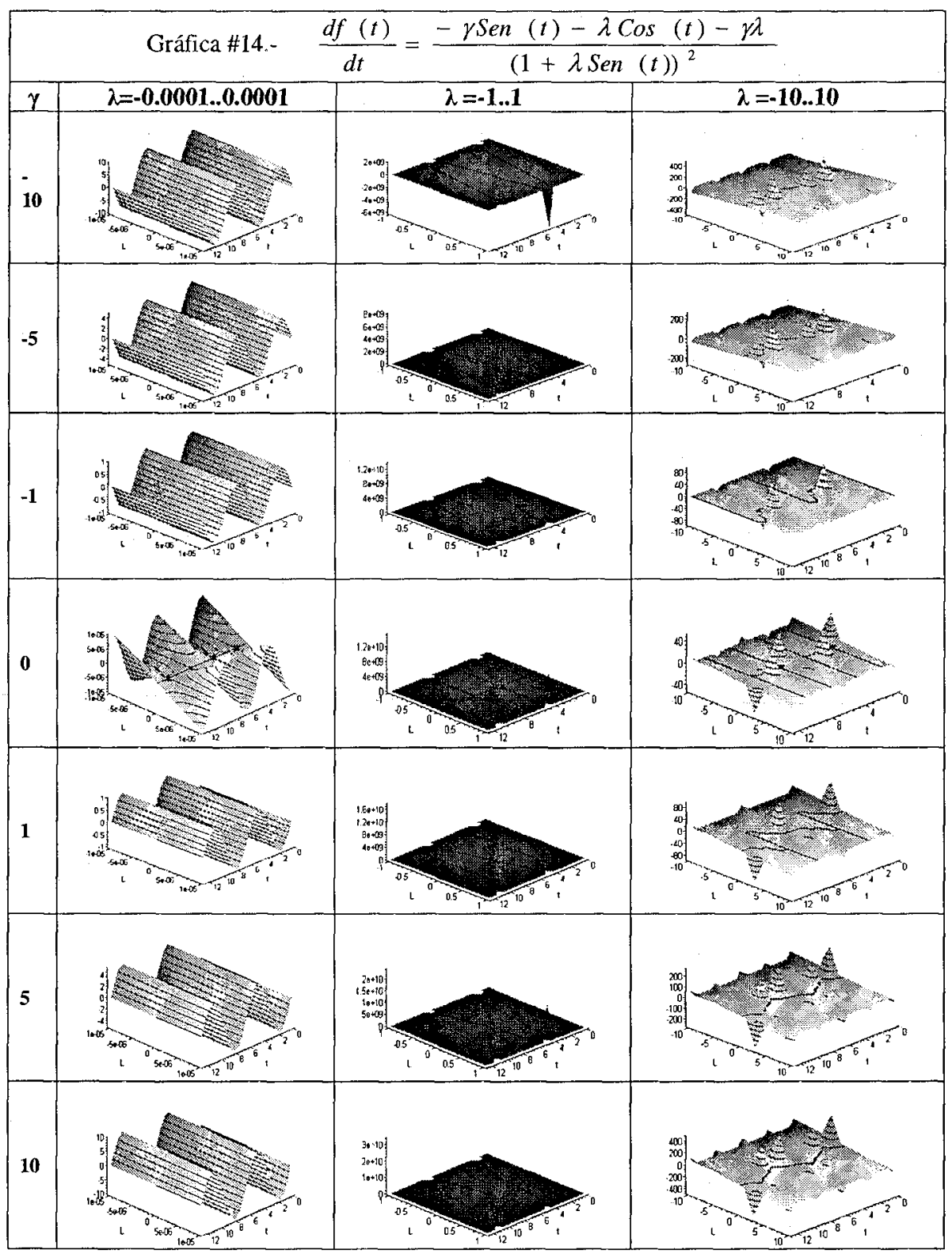




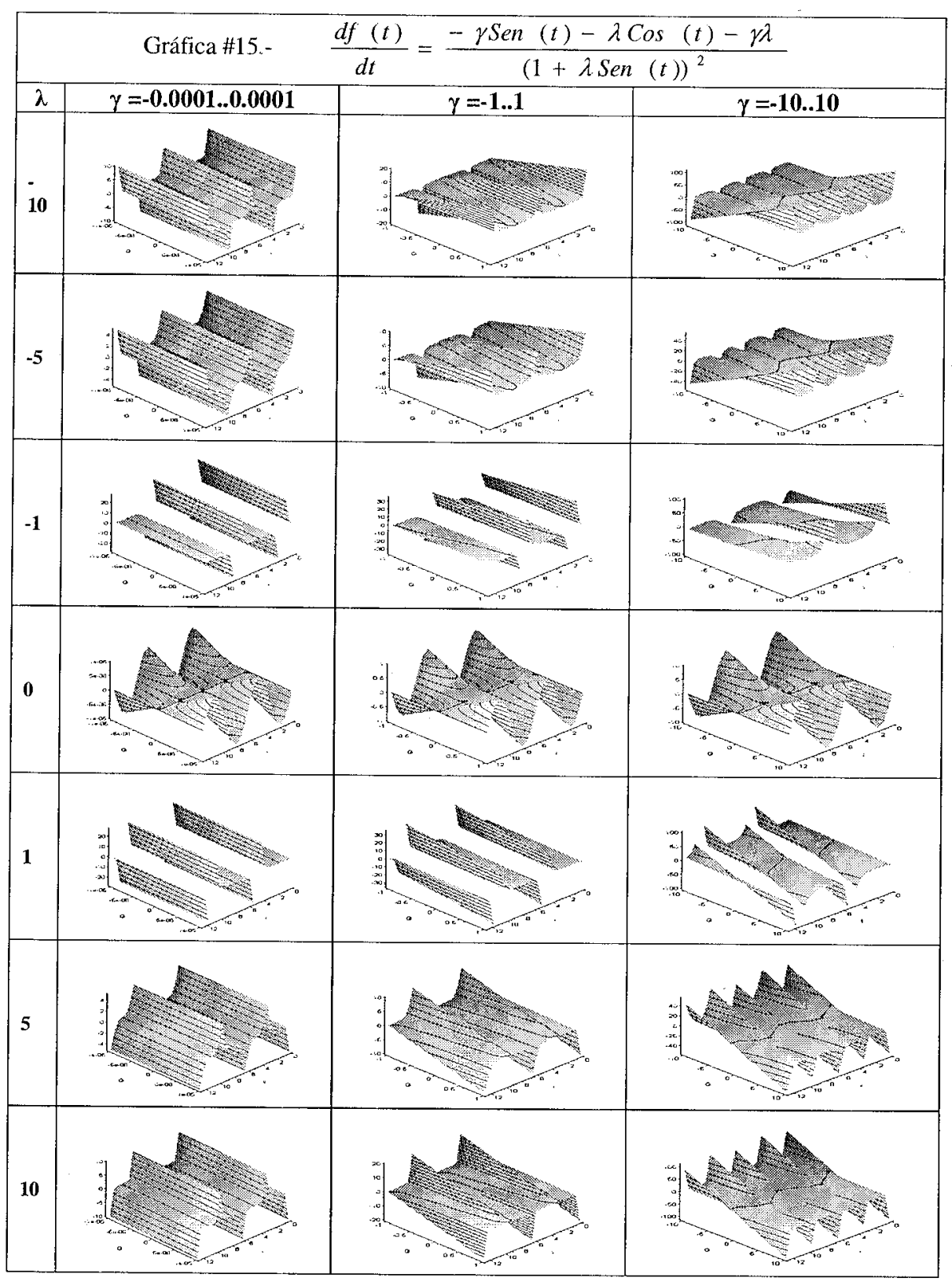

